Supporting Information for the Paper Entitled:

\title{
Side-On Coordination of Nitrous Oxide to a Mononuclear Cobalt Center
}

Charles C. Mokhtarzadeh, Chinglin Chan, Curtis E. Moore, Arnold L. Rheingold, Joshua S. Figueroa*

Department of Chemistry and Biochemistry, University of California, San Diego, 9500 Gilman Dr. Mail Code 0358, La Jolla, California 92093-0358.

\section{Email:jsfig@ucsd.edu}

\section{Contents:}

S1. Synthetic Procedures................................................................... 2

S2. Selected FTIR Spectra

S3. Selected NMR Spectra......$\ldots \ldots \ldots \ldots \ldots \ldots \ldots \ldots \ldots \ldots \ldots \ldots \ldots \ldots \ldots \ldots \ldots \ldots \ldots \ldots \ldots \ldots$

S4. Computational Results ..................................................... 18

S5. Crystallographic Structure Determinations ............................. 27

S6. References................................................................................ 34 


\section{S1. Synthetic Procedures}

S1.1. General Considerations: All manipulations were carried out under an atmosphere of dry dinitrogen or Argon using standard schlenk, and glove box techniques. Solvents were dried and degassed according to standard procedures. ${ }^{1}$ Unless otherwise stated all materials were obtained from commercial vendors and used as received or purified by standard procedures. ${ }^{2}$ Benzene- $d_{6}$ and Toluene- $d_{8}$ were dried with $\mathrm{Na} / \mathrm{K}$ and Benzophenone followed by distillation and stored on $4 \AA$ molecular sieves for 3 days prior to use. Celite 405 (Fischer Scientific) was dried under vacuum for 24 hours at a temperature greater then $250^{\circ} \mathrm{C}$ and stored inside the glove box prior to use. ${ }^{15} \mathrm{~N}_{2} \mathrm{O}(99.2 \%)$ was purchased from ICON Stable Isotopes and used as received. $\mathrm{KC}_{8}$ was prepared according to published procedures. ${ }^{3}$ The $m$-terphenyl isocyanide $\mathrm{CNAr}^{\mathrm{Tripp} 2}{ }^{4}$ and $\mathrm{Cp}^{*} \mathrm{CoI}_{2}(\mathrm{CO})^{5}$ were prepared according to literature procedures.

Solution ${ }^{1} \mathrm{H},{ }^{13} \mathrm{C}\left\{{ }^{1} \mathrm{H}\right\}$, and ${ }^{15} \mathrm{~N}$ NMR spectra were recorded on a Varian Mercury 400 spectrometer, a Bruker Advance 300 spectrometer, a Varian XSENS-500 spectrometer, and a Joel ECA 500 spectrometer. ${ }^{1} \mathrm{H}$, and ${ }^{13} \mathrm{C}$ chemical shifts are reported in ppm relative to $\mathrm{SiMe}_{4}\left({ }^{1} \mathrm{H}\right.$ and $\left.{ }^{13} \mathrm{C} \delta=0.0 \mathrm{ppm}\right)$ with reference to residual solvent references of 7.16 ppm $\left({ }^{1} \mathrm{H}\right)$ and $128.06 \mathrm{ppm}\left({ }^{13} \mathrm{C}\right)$ for benzene- $d_{6}{ }^{5}$, and $2.08 \mathrm{ppm}\left({ }^{1} \mathrm{H}\right)$, and $20.4 \mathrm{ppm}\left({ }^{13} \mathrm{C}\right)$ for cyclohexane- $d{ }^{12} .{ }^{5}{ }^{15} \mathrm{~N}$ chemical shifts were externally referenced to $\left({ }^{15} \mathrm{NH}_{4}\right)_{2} \mathrm{SO}_{4}$ in $\mathrm{H}_{2} \mathrm{O}$ and calibrated to $\mathrm{NH}_{3(l)}(\delta=0.0 \mathrm{ppm}) .{ }^{6}{ }^{31} \mathrm{P}$ chemical shifts were externally referenced to $85 \% \mathrm{H}_{3} \mathrm{PO}_{4}(\delta=0.0 \mathrm{ppm})$. FTIR spectra were recorded on a Thermo-Nicolet iS 10 FTIR spectrometer. Samples were prepared either as $\mathrm{KBr}$ pellets, or a $\mathrm{C}_{6} \mathrm{D}_{6}$, solution injected into a Thermo Fischer solution cell equipped with $\mathrm{KBr}$ windows. For solution FTIR spectra, solvent peaks were digitally subtracted from all spectra by comparisons with an authentic spectrum obtained immediately prior to that of the sample. In situ FTIR measurements were recorded with a Metler Toledo ReactIR 45M utilizing a SiComp tipped probe. For in situ FTIR spectral measurements solvent peaks were digitally subtracted from all spectra by comparisons with an authentic spectrum obtained immediately prior data collection. In situ FTIR spectra were prepared as solutions with a concentration of $0.05 \mathrm{M}$ or higher to maximize signal to noise. The following abbreviations were used for the intensities and characteristic of important IR absorption bands: vs = very strong, $\mathrm{s}=$ strong, $\mathrm{m}=$ medium, $\mathrm{w}=$ weak, $\mathrm{vw}=$ very weak, $\mathrm{b}=$ broad, $\mathrm{vb}=$ very broad, $\mathrm{sh}=$ shoulder. Combustion analyses were performed by Midwest Microlab LLC, Indianapolis, IN

S1.2. Synthesis of $\mathbf{C p}^{*} \mathbf{C o I}_{2} \mathbf{C N A r}{ }^{\text {Tripp2 }}$ : To a $\mathrm{C}_{6} \mathrm{H}_{6}$ solution $(60 \mathrm{~mL})$ of $\mathrm{Cp}^{*} \mathrm{CoI}_{2} \mathrm{CO}(1.55$ g, 3.26 mmoles, 1 equiv) was added CNAr ${ }^{\text {Tripp2 }}$ (1.89 g, 3.32 mmoles, 1.02 equiv) all at once. Upon addition there was an immediate effervescence indicative of CO loss. The mixture was allowed to stir for $c a$. 4 hours where upon all volatiles were removed in vacuo. There after, the resulting purple solid was taken up in $n$-Pentane and filtered over a medium glass sintered frit to yield $\mathrm{Cp}^{*} \mathrm{CoI}_{2} \mathrm{CNAr}^{\text {Tripp2 }}$ as a purple solid, $3.05 \mathrm{~g}, 3.19$ mmoles, 98\% yield. X-ray Diffraction/Analytically pure crystals were grown from a $\mathrm{CH}_{2} \mathrm{Cl}_{2}$ solution layered with $n$-Hexane (1:6) over one week. ${ }^{1} \mathrm{H}$ NMR (400.1 MHz, $\left.\mathrm{C}_{6} \mathrm{D}_{6}, 20{ }^{\circ} \mathrm{C}\right): \delta=7.26$ (s, 4H, m-Trip), 7.02 (d, 2H, 8Hz, m-Ph), 6.84 (t, 1H, 8Hz, p-ph), 2.91 (sept, 2H, 7Hz, $\left.\mathrm{CH}\left(\mathrm{CH}_{3}\right)_{2}\right), 2.85$ (sept, 4H, 7Hz, $\left.\mathrm{CH}\left(\mathrm{CH}_{3}\right)_{2}\right), 1.60$ (s, 15H, H-Cp*), 1.57 (d, 12H, $7 \mathrm{~Hz}$, $\left.\mathrm{CH}\left(\mathrm{CH}_{3}\right)_{2}\right), 1.37\left(\mathrm{~d}, 12 \mathrm{H}, 7 \mathrm{~Hz}, \mathrm{CH}\left(\mathrm{CH}_{3}\right)_{2}\right), 1.12\left(\mathrm{~d}, 12 \mathrm{H}, 7 \mathrm{~Hz}, \mathrm{CH}\left(\mathrm{CH}_{3}\right)_{2}\right) \mathrm{ppm} .{ }^{13} \mathrm{C}\left\{{ }^{1} \mathrm{H}\right\}$ 
NMR (125.7 MHz, $\left.\mathrm{C}_{6} \mathrm{D}_{6}, 20^{\circ} \mathrm{C}\right): \delta=158.9(\mathrm{CNR}), 149.7,146.8,139.7,133.2,131.7,122.0$, 127.7, 96.5, 35.1, 31.4, 25.4, 24.7, 24.5, 11.4. FTIR ( $\mathrm{C}_{6} \mathrm{D}_{6}$; $\mathrm{KBr}$ Window; $\left.20{ }^{\circ} \mathrm{C}\right): v_{\mathrm{CN}}=$ 2130 (s), also 2960 (m), 2927 (w), 2905 (w), $2868(w), 1605$ (w), $1566(w), 1464(w), 1430$ (w), $1412(\mathrm{w}), 1382(\mathrm{w}), 1363(\mathrm{w}), 1204(\mathrm{w}), 1071(\mathrm{w}), 1054(\mathrm{w}), 1020(\mathrm{w}), 941(\mathrm{w}), 877$ (w), 759 (w), $681(\mathrm{w}) \mathrm{cm}^{-1}$. Anal. Calcd. For $\mathrm{C}_{47} \mathrm{H}_{64} \mathrm{NI}_{2} \mathrm{Co}$ : C, 59.04; H, 6.75; N, 1.47 . Found: C, 59.17; H, 6.80; N, 1.47.

S1.3. Synthesis of $\mathbf{C p} * \mathbf{C o}\left(\mathbf{N}_{2}\right) \mathbf{C N A r}{ }^{\text {Tripp2 }}$ (1): To a thawing $\mathrm{Et}_{2} \mathrm{O} / \mathrm{DME}$ solution $(2: 1,9$ $\mathrm{mL}$ ) of $\mathrm{Cp}^{*} \mathrm{CoI}_{2} \mathrm{CNAr}^{\text {Dipp2 }}$ (0.627 g, 0.656 mmoles, 1 equiv) $\mathrm{KC}_{8}(0.189 \mathrm{~g}, 1.40$ mmoles, 2.1 equiv) was added all at once. The reaction mixture was allowed to stir for $c a .20$ minutes where upon a color change from purple to yellow/brown was observed. Upon completion the reaction mixture was filtered over a medium porosity glass sintered frit packed with Celite to remove $\mathrm{C}_{8}$ and $\mathrm{KI}$ followed by removal of all volatiles in vacuo. Upon concentration to a solid the resulting brown residue was taken up in $n$-Pentane (20 $\mathrm{mL}$ ) and allowed to stir for 10 minutes at room temperature followed by evaporation to a solid to desolvate any residual KI. This procedure was repeated an additional two times. Following concentration to a solid the brown solid was taken up in $\mathrm{C}_{6} \mathrm{H}_{6}$ and filtered over Celite packed on fiberglass followed by lyophilization to a solid. The resulting solid was then recrystallize from $\mathrm{Et}_{2} \mathrm{O}(1 \mathrm{~mL})$ with 2 drops of $\mathrm{C}_{6} \mathrm{H}_{6}$ over 36 hours to yield $\mathrm{Cp}^{*} \mathrm{Co}\left(\mathrm{N}_{2}\right) \mathrm{CNAr}^{\text {Tripp} 2}$ as dark brown plates $0.298 \mathrm{~g}, 0.408$ mmoles, $62 \%$ yield. ${ }^{1} \mathrm{H}$ NMR (499.8 MHz, $\mathrm{C}_{6} \mathrm{D}_{6}, 20{ }^{\circ} \mathrm{C}$ ): $\delta=7.23$ (s, 4H, $m$-Tripp), 6.99 (d, 2H, $\left.7 \mathrm{~Hz}, m-\mathrm{Ph}\right), 6.94$ (d, 2H, 8Hz, $m-\mathrm{Ph}$ ), 6.90 (t, 1H, $7 \mathrm{~Hz}, o-\mathrm{Ph}$ ), 2.95 (sept., 4H, $\left.8 \mathrm{~Hz}, \mathrm{CH}\left(\mathrm{CH}_{3}\right)_{2}\right), 2.89$ (sept., $\left.2 \mathrm{H}, 7 \mathrm{~Hz}, \mathrm{CH}\left(\mathrm{CH}_{3}\right)_{2}\right), 1.49$ (d, 12H, 7Hz, $\left.\mathrm{CH}\left(\mathrm{CH}_{3}\right)_{2}\right), 1.44\left(\mathrm{~s}, 15 \mathrm{H}, \mathrm{Cp}^{*}-\mathrm{H}\right), 1.32(\mathrm{~d}, 12 \mathrm{H}$, $\left.7 \mathrm{~Hz}, \mathrm{CH}\left(\mathrm{CH}_{3}\right)_{2}\right), 1.22\left(\mathrm{~d}, 12 \mathrm{H}, 7 \mathrm{~Hz}, \mathrm{CH}\left(\mathrm{CH}_{3}\right)_{2}\right) \mathrm{ppm} .{ }^{13} \mathrm{C}\left\{{ }^{1} \mathrm{H}\right\} \mathrm{NMR}\left(125.8 \mathrm{MHz}, \mathrm{C}_{6} \mathrm{D}_{6}\right.$, $\left.20{ }^{\circ} \mathrm{C}\right): \delta=179.7(C \mathrm{NR}), 148.9,146.8,136.5,134.2,131.2,130.0,124.2,121.3,92.6,34.9$, 31.4, 24.6, 24.4, 24.3, $10.2 \mathrm{ppm}$. FTIR $\left(\mathrm{C}_{6} \mathrm{D}_{6}\right.$; KBr Window; $\left.20{ }^{\circ} \mathrm{C}\right): v_{\mathrm{CN}}=2004(\mathrm{~s}), 2037$ $(\mathrm{m}), v_{\mathrm{NN}}=2111(\mathrm{~m})$, also $2961(\mathrm{~m}), 2929(\mathrm{w}), 2905(\mathrm{w}), 2868(\mathrm{w}), 1573(\mathrm{w}), 1463(\mathrm{w})$, 1412 (m), 1381 (w), 1362 (w), 1105 (w), 1070 (w), 1028 (w), $941(\mathrm{w}), 878$ (w), 745 (w) $\mathrm{cm}^{-1}$. Anal. Calcd. For $\mathrm{C}_{47} \mathrm{H}_{64} \mathrm{~N}_{3} \mathrm{Co}$ : C, 73.34; H, 8.34; N, 5.77. Found: C, 77.57; H, 8.49; N, 5.55 .

${ }^{1}$ H NMR Spectroscopy in Tol-ds: ${ }^{1} \mathrm{H}$ NMR $\left(400.1 \mathrm{MHz}\right.$, Tol- $\left.d_{8}, 20{ }^{\circ} \mathrm{C}\right): 7.17(\mathrm{~s}, 4 \mathrm{H}, \mathrm{m}$ Tripp), 6.93 (mult., 3H, m/p-Ph), 2.88 (mult., 6H, $\left.\mathrm{CH}\left(\mathrm{CH}_{3}\right)_{2}\right), 1.44(\mathrm{~d}, 12 \mathrm{H}, 7 \mathrm{~Hz}$, $\left.\mathrm{CH}\left(\mathrm{CH}_{3}\right)_{2}\right), 1.41\left(\mathrm{~s}, 15 \mathrm{H}, \mathrm{Cp}^{*}-\mathrm{H}\right), 1.30\left(\mathrm{~d}, 12 \mathrm{H}, 7 \mathrm{~Hz}, \mathrm{CH}\left(\mathrm{CH}_{3}\right)_{2}\right), 1.20(\mathrm{~d}, 12 \mathrm{H}, 7 \mathrm{~Hz}$, $\left.\mathrm{CH}\left(\mathrm{CH}_{3}\right)_{2}\right)$.

S1.4. Synthesis of $\mathrm{Cp} * \mathrm{Co}\left(\eta^{2}-N, N-N_{2} \mathrm{O}\right)\left(\mathrm{CNAr}^{\text {Tripp2 }}\right)$ (2): In a typical reaction $\mathrm{Cp}{ }^{*} \mathrm{Co}\left(\mathrm{N}_{2}\right)\left(\mathrm{CNAr}^{\text {Tripp2}}\right)$ (0.049 g, 0.067 mmoles, 1 equiv) was dissolved in $\mathrm{Et}_{2} \mathrm{O}(\sim 1 \mathrm{~mL})$ and transferred to a $25 \mathrm{~mL}$ re-sealable ampule in a $\mathrm{N}_{2(\mathrm{~g})}$ filled glove box, and then brought out of the glove box and connected to the schlenk line. There after the solution was subject to one freeze pump thaw cycle (FPT) and then cooled to $-78{ }^{\circ} \mathrm{C}$ and allowed to equilibrate for 10 mins. The mixture was then placed under an atmosphere of $\mathrm{N}_{2} \mathrm{O}_{(\mathrm{g})}$ and allowed to warm to $-25^{\circ} \mathrm{C}$ and react for 20 mins. The reaction mixture was then cooled back to -78 ${ }^{\circ} \mathrm{C}$ and rapidly transferred back into a $\mathrm{N}_{2}$ (g) filled glove box and placed in the freezer at $40{ }^{\circ} \mathrm{C}$. Storage at $-40{ }^{\circ} \mathrm{C}$ for 24 hours afforded yellow crystals that were suitable for X-ray diffraction, $0.020 \mathrm{~g}, 0.027$ mmoles, $40 \%$ yield. ${ }^{1} \mathrm{H}$ NMR $\left(499.9 \mathrm{MHz}\right.$, Tol- $\left.d_{8},-20{ }^{\circ} \mathrm{C}\right)$ : 
7.22 (s, 2H, m-Tripp), 7.20 (s, 2H m-Tripp), 6.88 (d, 2H, $7 \mathrm{~Hz}, m$-Ph), 6.82 (t, 1H, $7 \mathrm{~Hz}, p$ $\mathrm{Ph}$ ), 2.94 (sept., 2H, $\left.7 \mathrm{~Hz}, \mathrm{CH}\left(\mathrm{CH}_{3}\right)_{2}\right), 2.70$ (sept., 4H, $\left.7 \mathrm{~Hz}, \mathrm{CH}\left(\mathrm{CH}_{3}\right)_{2}\right), 1.46(\mathrm{~d}, 6 \mathrm{H}, 7 \mathrm{~Hz}$, $\left.\mathrm{CH}\left(\mathrm{CH}_{3}\right)_{2}\right), 1.43\left(\mathrm{~d}, 6 \mathrm{H}, 7 \mathrm{~Hz}, \mathrm{CH}\left(\mathrm{CH}_{3}\right)_{2}\right), 1.41\left(\mathrm{dd}, 12 \mathrm{H}, 5 \mathrm{~Hz}, 3 \mathrm{~Hz}, \mathrm{CH}\left(\mathrm{CH}_{3}\right)_{2}\right) 1.32(\mathrm{~s}$, $\left.15 \mathrm{H}, \mathrm{Cp}^{*}-\mathrm{H}\right), 1.16\left(\mathrm{t}, 12 \mathrm{H}, 7 \mathrm{~Hz}, \mathrm{CH}\left(\mathrm{CH}_{3}\right)_{2}\right) .{ }^{13} \mathrm{C}\left\{{ }^{1} \mathrm{H}\right\} \mathrm{NMR}\left(125.8 \mathrm{MHz}, \mathrm{Tol}-d_{8},-20{ }^{\circ} \mathrm{C}\right)$ : $\delta=166.4(C \mathrm{NR}), 149.5,146.0,145.9,146.8,138.8,132.4,129.8,127.1,121.6,121.5,94.9$, 35.1, 31.4, 31.3, 24.8, 24.4, 24.5, 24.1, 9.2 ppm. ${ }^{15} \mathrm{~N}\left\{{ }^{1} \mathrm{H}\right\}$ NMR $\left(50.7 \mathrm{MHz}\right.$, Tol- $d_{8},-20$ $\left.{ }^{\circ} \mathrm{C}\right): 308.6(\mathrm{~d}, 17 \mathrm{~Hz}), 159.2(\mathrm{~d}, 17 \mathrm{~Hz})$. (Due to the thermal sensitivity and its propensity to decompose at temperatures greater than $0{ }^{\circ} \mathrm{C}$ elemental analysis was not obtained. IR spectroscopy is presented below in Section 2.

S1.5. Synthesis of $\mathrm{Cp} * \mathrm{Co}\left(\eta^{2}-\mathrm{N}, \mathrm{O}-\mathrm{o}-\mathrm{CH}_{3}-\mathrm{C}_{6} \mathrm{H}_{4} \mathrm{NO}\right)\left(\mathrm{CNAr}{ }^{\text {Tripp2 }}\right)$ (3): To a solution of $\mathrm{Cp}^{*} \mathrm{Co}\left(\mathrm{N}_{2}\right) \mathrm{CNAr}^{\text {Tripp} 2}\left(0.052 \mathrm{~g}, 0.071 \mathrm{mmoles}, 1\right.$ equivs) in $1 \mathrm{~mL}^{\circ} \mathrm{C}_{6} \mathrm{D}_{6}$ a solution of $o$ Tol-Nitroso-benzene ( $0.009 \mathrm{~g}, 0.071 \mathrm{mmoles}, 1$ equivs) in $0.5 \mathrm{~mL}$ of $\mathrm{C}_{6} \mathrm{D}_{6}$ was added in one portion. Upon addition an immediate color change from brown to forest green was observed. Analysis via ${ }^{1} \mathrm{H}$ NMR indicated complete conversion to a new diamagnetic product. The resulting solution was concentrated to a solid under reduced pressure and recrystallized from a saturated solution of $n$-pentane and $\mathrm{TMS}_{2} \mathrm{O}(1: 5 ; 2 \mathrm{~mL})$ at $-40{ }^{\circ} \mathrm{C}$ over 1 week to afford analytically pure forest green crystals of $\mathrm{Cp}^{*} \mathrm{Co}\left(\eta^{2}-N, O-\right.$ $\left.\mathrm{C}_{7} \mathrm{H}_{7} \mathrm{NO}\right) \mathrm{CNAr}^{\text {Tripp2}} \bullet \mathrm{TMS}_{2} \mathrm{O} 0.031 \mathrm{~g}, 0.033$ mmoles, $45 \%$ yield. ${ }^{1} \mathrm{H}$ NMR $(499.8 \mathrm{MHz}$, $\mathrm{C}_{6} \mathrm{D}_{6}, 20^{\circ} \mathrm{C}$ ): 7.24 (bd, $1 \mathrm{H}, 10 \mathrm{~Hz}, m$-Tol), 7.22 (s, 4H, $m$-Tripp), 7.10 (d, 1H, 7Hz, $m$-Tol), 7.03 (t, 1H, 6Hz, $m$-Tol), 6.98 (t, 1H, 8 Hz, $p$-Tol), 6.90 (d, 2H, 8Hz, $m$-Tripp), 6.81 (t, 1H, $8 \mathrm{~Hz}, p$-Tripp), 2.88 (sept., 2H, $\left.7 \mathrm{~Hz}, \mathrm{CH}\left(\mathrm{CH}_{3}\right)_{2}\right), 2.79$ (sept., 4H, $\left.7 \mathrm{~Hz}, \mathrm{CH}\left(\mathrm{CH}_{3}\right)_{2}\right), 2.12$ (s, 3H, Me-Tol), 1.49 (bd, 12H, 6Hz, $\left.\mathrm{CH}\left(\mathrm{CH}_{3}\right)_{2}\right), 1.31$ (d, 12H, 7Hz, $\left.\mathrm{CH}\left(\mathrm{CH}_{3}\right)_{2}\right), 1.15$ (d, $\left.12 \mathrm{H}, 7 \mathrm{~Hz}, \mathrm{CH}\left(\mathrm{CH}_{3}\right)_{2}\right), 1.13\left(\mathrm{~s}, 15 \mathrm{H}, \mathrm{Cp}^{*}-\mathrm{H}\right) .{ }^{13} \mathrm{C}\left\{{ }^{1} \mathrm{H}\right\} \mathrm{NMR}\left(125.8 \mathrm{MHz}, \mathrm{C}_{6} \mathrm{D}_{6}, 20{ }^{\circ} \mathrm{C}\right): \delta$ $=166.3(C \mathrm{NR}), 162.5,149.3,146.9,139.1,133.3,131.0,130.8,130.0,129.1,127.0,121.6$, $126.4,121.6,121.5,115.1,93.8,34.9,31.5,24.8,24.4,24.2,19.6,9.4$ ppm. FTIR $\left(\mathrm{C}_{6} \mathrm{D}_{6}\right.$; $\mathrm{KBr}$ Window; $\left.20{ }^{\circ} \mathrm{C}\right): v_{\mathrm{CN}}=2099(\mathrm{~s})$, also $2961(\mathrm{~m}), 2927(\mathrm{w}), 2906(\mathrm{w}), 2869(\mathrm{w}), 1587$ (w), 1464 (w), 1414 (w), 1382 (w), 1363 (w), 1254 (w), $1201(\mathrm{w}), 1108(\mathrm{w}), 1071(\mathrm{w})$, 1053(w), $1029(\mathrm{w}), 940(\mathrm{w}), 879(\mathrm{w}), 756(\mathrm{w}), 719$ (w) $\mathrm{cm}^{-1}$. Anal. Calcd. For $\mathrm{C}_{54} \mathrm{H}_{71} \mathrm{~N}_{2} \mathrm{OCo}$ : C, 78.79; H, 8.70; N, 3.41. Found: C, 77.23; H, 8.37; N, 3.41.

S1.6. Independent Synthesis of $\mathrm{Cp}^{*} \mathrm{Co}(\mathrm{OH})\left(\mathrm{CNAr}^{\text {Tripp2 }}\right)$ (4): To a solution of $\mathrm{Cp}^{*} \mathrm{Co}\left(\mathrm{N}_{2}\right)\left(\mathrm{CNAr}^{\text {Tripp} 2}\right)(0.053 \mathrm{~g}, 0.0721$ mmoles, 1 equiv. $)$ in $2 \mathrm{~mL}$ of THF a solution of $\mathrm{Cp}^{*} \mathrm{CoI}_{2}\left(\mathrm{CNAr}^{\text {Tripp} 2}\right)(0.068 \mathrm{~g}, 0.0721$ mmoles, 1 equiv. $)$ in $1 \mathrm{~mL}$ of THF was added dropwise over the course of $1 \mathrm{~min}$. An instant color change to deep orange was observed and solution FTIR analysis of the reaction mixture displayed a prominent isocyanide shift at $2089 \mathrm{~cm}^{-1}$ (THF) intermediate in intensity to both starting materials and indication of the in situ formation of $\mathrm{Cp}^{*} \mathrm{CoI}\left(\mathrm{CNAr}^{\text {Tripp2}}\right)$. There after a THF solution containing $\mathrm{NaOH}$ (0.009 g, 0.216 mmoles, 3 equiv.) and 18-crown-6 (0.036 g, 0.144 mmols, 2 equiv.) were added and the solution was allowed to stir at room temperature for 24 hours. After 24 hours a color change from deep orange to dark red was observed. The solution was concentrated to an oil and then slurried in $n$-pentane $(4 \mathrm{~mL})$ and evaporated to a solid to desolvate any remaining $\mathrm{NaOH} / \mathrm{NaI} / 18$-crown-6. This was repeated two additional times. There after the resultant solid was taken up in $n$-pentane and filtered over Celite packed on fiberglass $(2 \mathrm{~cm})$ and concentrated to $c a .1 \mathrm{~mL}$ and placed in the freezer for recrystallization at $-40{ }^{\circ} \mathrm{C}$ for 7 days to afford analytically pure red crystals of $\mathrm{Cp}^{*} \mathrm{Co}(\mathrm{OH})\left(\mathrm{CNAr}{ }^{\mathrm{Tripp} 2}\right)$, 
$0.040 \mathrm{~g}, 0.056$ mmoles, $39 \%$. Analysis via ${ }^{1} \mathrm{H}$ NMR indicated paramagnetically shifted resonances. ${ }^{1} \mathrm{H}$ NMR analysis indicated that $\mathrm{Cp}^{*} \mathrm{Co}(\mathrm{OH})\left(\mathrm{CNAr}^{\text {Tripp2}}\right)$ does not have any distinguishable NMR features. Evans method magnetic moment determination based on an average of 5 measurements: $1.72 \mu_{\mathrm{B}}$ consistent with a spin $\frac{1}{2}$ system. FTIR $\left(\mathrm{C}_{6} \mathrm{D}_{6} ; \mathrm{KBr}\right.$ Window; $\left.20{ }^{\circ} \mathrm{C}\right): v_{\mathrm{CN}}=2059(\mathrm{~s}), 2013(\mathrm{w}, \mathrm{sh}), v_{\mathrm{OH}}=3607$ also $2959(\mathrm{~s}), 2925(\mathrm{w}), 2869$ (w), $2850(w), 1604(w), 1569(w), 1413(w), 1382(w), 1363(w), 1106(w), 1071(w), 990$ (w), 940 (w), 879 (w), 757 (w), 596 (w) cm ${ }^{-1}$. Anal. Calcd. For $\mathrm{C}_{47} \mathrm{H}_{65} \mathrm{NOCo}$ : C, 78.52; H, 9.11; N, 1.95. Found: C, 78.41; H, 8.99; N, 1.85 .

Solution FTIR spectroscopy in THF: FTIR (THF; KBr Window; $20{ }^{\circ} \mathrm{C}$ ): $v_{\mathrm{CN}}=2061(\mathrm{~s})$, $2014(\mathrm{w}, \mathrm{sh}), v_{\mathrm{OH}}=3608$ also $1605(\mathrm{w}), 1570(\mathrm{w}), 1412(\mathrm{w}), 1384(\mathrm{w}) \mathrm{cm}^{-1}$.

S1.7. Independent Synthesis OCNAr ${ }^{\text {Tripp2 }}$ : The isocyanate (OCNR) was prepared according to a modified literature procedure. ${ }^{7}$ Under an inert atmosphere a $\mathrm{CH}_{2} \mathrm{Cl}_{2}$ solution $(4 \mathrm{~mL})$ containing DMSO $\left(27 \mu \mathrm{L}, 0.388\right.$ mmoles, 1.1 equiv) was added $\mathrm{CNAr}^{\mathrm{Tripp} 2}(0.200$ g, $0.352,1$ equiv) as a solid. The reaction mixture was then cooled to $-60^{\circ} \mathrm{C}$ and allowed to equilibrate for 10 minutes where upon Trifluroaceticanhydride $(0.004 \mathrm{~g}, 0.018$ mmoles, 5 mol \%) was added all at once. The reaction was then allowed to warm to room temperature and react for 2 hours where upon a color change from colorless to yellow was observed. The resulting reaction mixture was then concentrated to a solid using rotary evaporation and then passed through a plug of Silica and eluted with a 3\% EtOAc/Hexanes solution, which was then re-concentrated to a solid to afford OCNAr ${ }^{\text {Tripp2 } 2}$ as a white solid $0.180 \mathrm{~g}, 0.344$ mmoles, $89 \%$. ${ }^{1} \mathrm{H}$ NMR $\left(499.9 \mathrm{MHz}\right.$, Tol- $d_{8},-20^{\circ} \mathrm{C}$ ): 7.22 (s, $2 \mathrm{H}, m$-Tripp), 7.20 (s, 2H m-Tripp), 6.88 (d, 2H, $7 \mathrm{~Hz}, m$-Ph), 6.82 (t, 1H, $7 \mathrm{~Hz}, p$-Ph), 2.94 (sept., $2 \mathrm{H}, 7$ $\left.\mathrm{Hz}, \mathrm{CH}\left(\mathrm{CH}_{3}\right)_{2}\right), 2.70$ (sept., $\left.4 \mathrm{H}, 7 \mathrm{~Hz}, \mathrm{CH}\left(\mathrm{CH}_{3}\right)_{2}\right), 1.46$ (d, 6H, 7Hz, $\left.\mathrm{CH}\left(\mathrm{CH}_{3}\right)_{2}\right), 1.43$ (d, $\left.6 \mathrm{H}, 7 \mathrm{~Hz}, \mathrm{CH}\left(\mathrm{CH}_{3}\right)_{2}\right), 1.41\left(\mathrm{dd}, 12 \mathrm{H}, 5 \mathrm{~Hz}, 3 \mathrm{~Hz}, \mathrm{CH}\left(\mathrm{CH}_{3}\right)_{2}\right) 1.32\left(\mathrm{~s}, 15 \mathrm{H}, \mathrm{Cp}^{*}-\mathrm{H}\right), 1.16$ (t, $\left.12 \mathrm{H}, 7 \mathrm{~Hz}, \mathrm{CH}\left(\mathrm{CH}_{3}\right)_{2}\right) .{ }^{13} \mathrm{C}\left\{{ }^{1} \mathrm{H}\right\} \mathrm{NMR}\left(125.8 \mathrm{MHz}, \mathrm{C}_{6} \mathrm{D}_{6},-20{ }^{\circ} \mathrm{C}\right): \delta=149.8,147.3,137.2$, 134.3, 133.6, 130.0, 127.1, $125.3(\mathrm{O}=C=\mathrm{N}), 121.4,34.9,31.3,24.7,24.3,24.1 \mathrm{ppm}$. FTIR $\left(\mathrm{C}_{6} \mathrm{H}_{6} ; \mathrm{KBr}\right.$ Window; $\left.20{ }^{\circ} \mathrm{C}\right): v_{\mathrm{OCN}}=2278(\mathrm{~m}), 2242(\mathrm{~s})$, also $2963(\mathrm{~m}), 2929(\mathrm{w}), 2869$ (w), 1605 (w), 1566 (w), 1433 (w), 1384 (w), 1364 (w), 1073 (w), 879 (w), 860 (w), 803 (w), $758(\mathrm{w}), 522(\mathrm{w}) \mathrm{cm}^{-1}$. HRMS (ESI-APCI-TOF, pos. ion; MeCN) $\mathrm{m} / \mathrm{z}$ calcd for $\left[\mathrm{C}_{37} \mathrm{H}_{50} \mathrm{NOH}\right]^{+}: 534.3887$. Found for $\left[\mathrm{C}_{37} \mathrm{H}_{50} \mathrm{NOH}\right]^{+}: 524.3883, \delta=-0.8$.

${ }^{1} \mathrm{H}$ NMR Spectroscopy in Tol-d $\boldsymbol{d}_{8}:{ }^{1} \mathrm{H}$ NMR $\left(499.9 \mathrm{MHz}\right.$, Tol- $\left.d_{8}, 20{ }^{\circ} \mathrm{C}\right): 7.19(\mathrm{~s}, 4 \mathrm{H}, \mathrm{m}-$ Tripp), 7.03 (d, 2H, $8 \mathrm{~Hz}, m$-Ph), 6.96 (t, 1H, $8 \mathrm{~Hz}, p$-Ph), 2.80 (sept., $6 \mathrm{H}, 7 \mathrm{~Hz}, \mathrm{CH}\left(\mathrm{CH}_{3}\right)_{2}$ ), $1.28\left(\mathrm{~d}, 12 \mathrm{H}, 7 \mathrm{~Hz}, \mathrm{CH}\left(\mathrm{CH}_{3}\right)_{2}\right), 1.25\left(\mathrm{~d}, 12 \mathrm{H}, 7 \mathrm{~Hz}, \mathrm{CH}\left(\mathrm{CH}_{3}\right)_{2}\right), 1.17(\mathrm{~d}, 12 \mathrm{H}, 7 \mathrm{~Hz}$, $\left.\mathrm{CH}\left(\mathrm{CH}_{3}\right)_{2}\right) .{ }^{13} \mathrm{C}\left\{{ }^{1} \mathrm{H}\right\}$ NMR $\left(125.8 \mathrm{MHz}\right.$, Tol- $\left.d_{8}, 20{ }^{\circ} \mathrm{C}\right): \delta=149.6,147.2,137.2,134.3$, 133.6, 130.0, $127.0(\mathrm{O}=C=\mathrm{N}), 121.3,121.2,34.9,31.3,24.6,24.3,24.1 \mathrm{ppm}$.

Solution FTIR Spectroscopy in Toluene: FTIR (Toluene; $\mathrm{KBr}$ Window; $\left.20{ }^{\circ} \mathrm{C}\right)$ : $v_{\mathrm{OCN}}=$ 2276 (m), 2241 (s), also 1394 (w), 1385 (w), 1363 (w), 1239 (w), 942 (w), 878 (w), 802 (w), $653 \mathrm{~cm}^{-1}$.

Solution FTIR Spectroscopy in THF: FTIR (THF; KBr Window; $\left.20{ }^{\circ} \mathrm{C}\right): v_{\mathrm{OCN}}=2276$ (m), 2242 (s), also 1607 (w), 1569 (w), $758(w) \mathrm{cm}^{-1}$. 
S1.8. Independent Synthesis of $\mathrm{Cp} * \mathrm{Co}\left(\mathrm{PPh}_{3}\right)\left(\mathrm{CNAr}^{\text {Tripp2 }}\right)$ (5): To a solution of $\mathrm{Cp}^{*} \mathrm{Co}\left(\mathrm{N}_{2}\right) \mathrm{CNAr}^{\text {Tripp2 }}$ (0.041 g, 0.056 mmoles, 1 equivs) in $1 \mathrm{~mL}$ of $\mathrm{C}_{6} \mathrm{D}_{6}$ a solution of $\mathrm{PPh}_{3}\left(0.015 \mathrm{~g}, 0.056\right.$ mmoles, 1 equivs) in $0.5 \mathrm{~mL}$ of $\mathrm{C}_{6} \mathrm{D}_{6}$ was added in one portion. Upon addition an immediate color change from brown to purple/black was observed. Analysis via ${ }^{1} \mathrm{H}$ NMR indicated complete conversion to a new diamagnetic product. The resulting solution was concentrated to a solid under reduced pressure and recrystallized from a supersaturated solution of $n$-pentane and $\mathrm{Et}_{2} \mathrm{O}(1: 5 ; 2 \mathrm{~mL})$ at $-40{ }^{\circ} \mathrm{C}$ over 1 week to afford analytically pure black crystals of $\mathrm{Cp}^{*} \mathrm{Co}\left(\mathrm{PPh}_{3}\right) \mathrm{CNAr}^{\text {Tripp2 }} 0.028 \mathrm{~g}, 0.029$ mmoles, $53 \%$ yield. ${ }^{1} \mathrm{H}$ NMR $\left(499.8 \mathrm{MHz}, \mathrm{C}_{6} \mathrm{D}_{6}, 20{ }^{\circ} \mathrm{C}\right): 7.66$ (t, $\left.6 \mathrm{H}, 8 \mathrm{~Hz}, \mathrm{P}-P h\right), 7.16$ (s, 4H, $m$-Tripp), $7.04(\mathrm{~d}, 2 \mathrm{H}, 8 \mathrm{~Hz}, m-\mathrm{Ph}), 7.00$ (m, 8H, P-Ph), 6.83 (t, 1H, $8 \mathrm{~Hz}, p$-Ph), 2.95 (sept., 6H, 7 $\left.\mathrm{Hz}, \mathrm{CH}\left(\mathrm{CH}_{3}\right)_{2}\right), 1.40$ (d, 12H, 7Hz CH$\left.\left(\mathrm{CH}_{3}\right)_{2}\right), 1.37$ (s, 15H, Cp-Me), $1.31(\mathrm{~d}, 12 \mathrm{H}, 7 \mathrm{~Hz}$, $\left.\mathrm{CH}\left(\mathrm{CH}_{3}\right)_{2}\right), 1.14\left(\mathrm{~d}, 12 \mathrm{H}, 7 \mathrm{~Hz}, \mathrm{CH}\left(\mathrm{CH}_{3}\right)_{2}\right), 1.09$ (broad singlet, $\left.15 \mathrm{H}, \mathrm{CH}\left(\mathrm{CH}_{3}\right)_{2}\right) .{ }^{13} \mathrm{C}\left\{{ }^{1} \mathrm{H}\right\}$ NMR (125.8 MHz, $\left.\mathrm{C}_{6} \mathrm{D}_{6}, 20{ }^{\circ} \mathrm{C}\right): \delta=192.1\left(\mathrm{CNR}, \mathrm{d},{ }^{13} \mathrm{C}-{ }^{31} \mathrm{P}\right.$ coupling = 34.6 Hz), 148.0, $147.0,138.5,138.2,137.0,135.9,134.5,134.4,134.3,134.1,133.9,131.9,128.9,128.8$, $127.5,127.4,123.1,121.3,92.4,34.7,31.2,26.1,24.4,23.1,10.8$ ppm. ${ }^{31} \mathrm{P}\left\{{ }^{1} \mathrm{H}\right\} \mathrm{NMR}$ $\left(121.4 \mathrm{MHz}, \mathrm{C}_{6} \mathrm{D}_{6}, 20{ }^{\circ} \mathrm{C}\right): \delta=66.7 \mathrm{ppm}$. FTIR $\left(\mathrm{C}_{6} \mathrm{D}_{6} ; \mathrm{KBr}\right.$ Window; $\left.20{ }^{\circ} \mathrm{C}\right): v_{\mathrm{CN}}=1919$ (s), also 3054 (w), 2961 (s), 2928 (m), 2904 (m), 2867 (m), $2118(\mathrm{w}), 2091(\mathrm{w}), 1605(\mathrm{w})$, $1570(\mathrm{w}), 1481(\mathrm{w}), 1461(\mathrm{w}), 1434(\mathrm{w}), 1407(\mathrm{w}), 1381(\mathrm{w}), 1362(\mathrm{w}), 1203(\mathrm{w}), 1086$ (w), 1069 (w), 1027 (w), $1000(\mathrm{w}), 943$ (w), 877 (w), 757 (w), $746(\mathrm{w}), 722(\mathrm{w}), 690(\mathrm{~m})$ $\mathrm{cm}^{-1}$. Anal. Calcd. For $\mathrm{C}_{63} \mathrm{H}_{82} \mathrm{NPCo}$ C, 80.22; H, 8.76; N, 1.48. Found: C, 80.55; H, 8.67; $\mathrm{N}, 1.50$.

S1.9. Independent Synthesis of $\mathrm{Cp}^{*} \mathrm{Co}\left(\eta^{2}-C, C-\mathrm{C}_{6} \mathrm{H}_{10}\right)\left(\mathrm{CNAr}{ }^{\text {Tripp2 }}\right)(6)$ : To a solution of $\mathrm{Cp}^{*} \mathrm{Co}\left(\mathrm{N}_{2}\right) \mathrm{CNAr}^{\text {Tripp2 }}\left(0.039 \mathrm{~g}, 0.054\right.$ mmoles, 1 equivs) in $1 \mathrm{~mL}$ of $\mathrm{C}_{6} \mathrm{D}_{6}$ a solution of $\mathrm{C}_{6} \mathrm{H}_{10}\left(0.0055 \mathrm{~mL}, 0.054\right.$ mmoles, 1 equivs) in $0.5 \mathrm{~mL}$ of $\mathrm{C}_{6} \mathrm{D}_{6}$ was added in one portion. Upon addition an immediate color change from brown to red/orange was observed. Analysis via ${ }^{1} \mathrm{H}$ NMR indicated complete conversion to a new diamagnetic product. The resulting solution was concentrated to a solid under reduced pressure and recrystallized from a saturated solution of $\mathrm{TMS}_{2} \mathrm{O} / n$-pentane $(1: 5 ; 1.5 \mathrm{~mL})$ at $-40{ }^{\circ} \mathrm{C}$ over 1 week to afford analytically pure black crystals of $\mathrm{Cp}{ }^{*} \mathrm{Co}\left(\eta^{2}-C, C-\mathrm{C}_{6} \mathrm{H}_{10}\right) \mathrm{CNAr}{ }^{\text {Tripp2 }} 0.017 \mathrm{~g}, 0.022 \mathrm{mmoles}$, $39.5 \%$ yield. ${ }^{1} \mathrm{H}$ NMR (499.8 MHz, $\mathrm{C}_{6} \mathrm{D}_{6}, 20{ }^{\circ} \mathrm{C}$ ): 7.23 (s, 4H, $8 \mathrm{~Hz}, m$-Tripp), 6.97 (d, $2 \mathrm{H}, 8 \mathrm{~Hz} m-\mathrm{Ph}), 6.81$ (t, 1H, $7 \mathrm{~Hz}, p-\mathrm{Ph}), 5.70$ (s, 2H, cyclohexene), 2.94 (overlapped sept., $\left.6 \mathrm{H}, \mathrm{CH}\left(\mathrm{CH}_{3}\right)_{2}\right), 2.24$ (broad mult., $2 \mathrm{H}$, cyclohexene), 2.00 (broad mult. $2 \mathrm{H}$, cyclohexene), 1.90 (broad mult., $2 \mathrm{H}$, cyclohexene), 1.50 (d, 12H, 7Hz, $\left.\mathrm{CH}\left(\mathrm{CH}_{3}\right)_{2}\right), 1.46$ (s, 15H, Cp-Me), $1.34\left(\mathrm{~d}, 12 \mathrm{H}, 7 \mathrm{~Hz}, \mathrm{CH}\left(\mathrm{CH}_{3}\right)_{2}\right), 1.31$ (overlapped broad mult., $2 \mathrm{H}$, cyclohexene), 1.14 (d, $\left.12 \mathrm{H}, 7 \mathrm{~Hz}, \mathrm{CH}\left(\mathrm{CH}_{3}\right)_{2}\right)$ ppm. ${ }^{13} \mathrm{C}\left\{{ }^{1} \mathrm{H}\right\} \mathrm{NMR}\left(125.8 \mathrm{MHz}, \mathrm{C}_{6} \mathrm{D}_{6}, 20{ }^{\circ} \mathrm{C}\right): \delta=175.0(\mathrm{CNR})$, $148.8,147.1,137.0,134.9,132.2,130.6,130.0,123.8,121.9,92.7,53.7,35.0,31.1,30.3$, 29.6, 25.7, 25.5, 24.3, 23.8, 23.7, 23.0, 9.6 ppm. FTIR $\left(\mathrm{C}_{6} \mathrm{D}_{6} ; \mathrm{KBr}\right.$ Window; $\left.20{ }^{\circ} \mathrm{C}\right): v_{\mathrm{CN}}$ $=1990(\mathrm{~s}), 2035(\mathrm{sh})$ also $2961(\mathrm{~s}), 2928(\mathrm{~m}), 2906(\mathrm{~m}), 2868(\mathrm{~m}), 2109(\mathrm{w}), 2091(\mathrm{w})$, 1465 (w), $1411(\mathrm{w}), 1382$ (w), 1363 (w), 1252 (w), 1240 (w), 1196 (w), 1104 (w), 1070 (w), 879 (w), $756(w) \mathrm{cm}^{-1}$. Anal. Calcd. For $\mathrm{C}_{53} \mathrm{H}_{74} \mathrm{NCo}$ : C, 81.19; H, 9.51; N, 1.79. Found: C, 78.70; H, 9.35; N, 1.78. 


\section{S2. Selected FTIR Spectra}

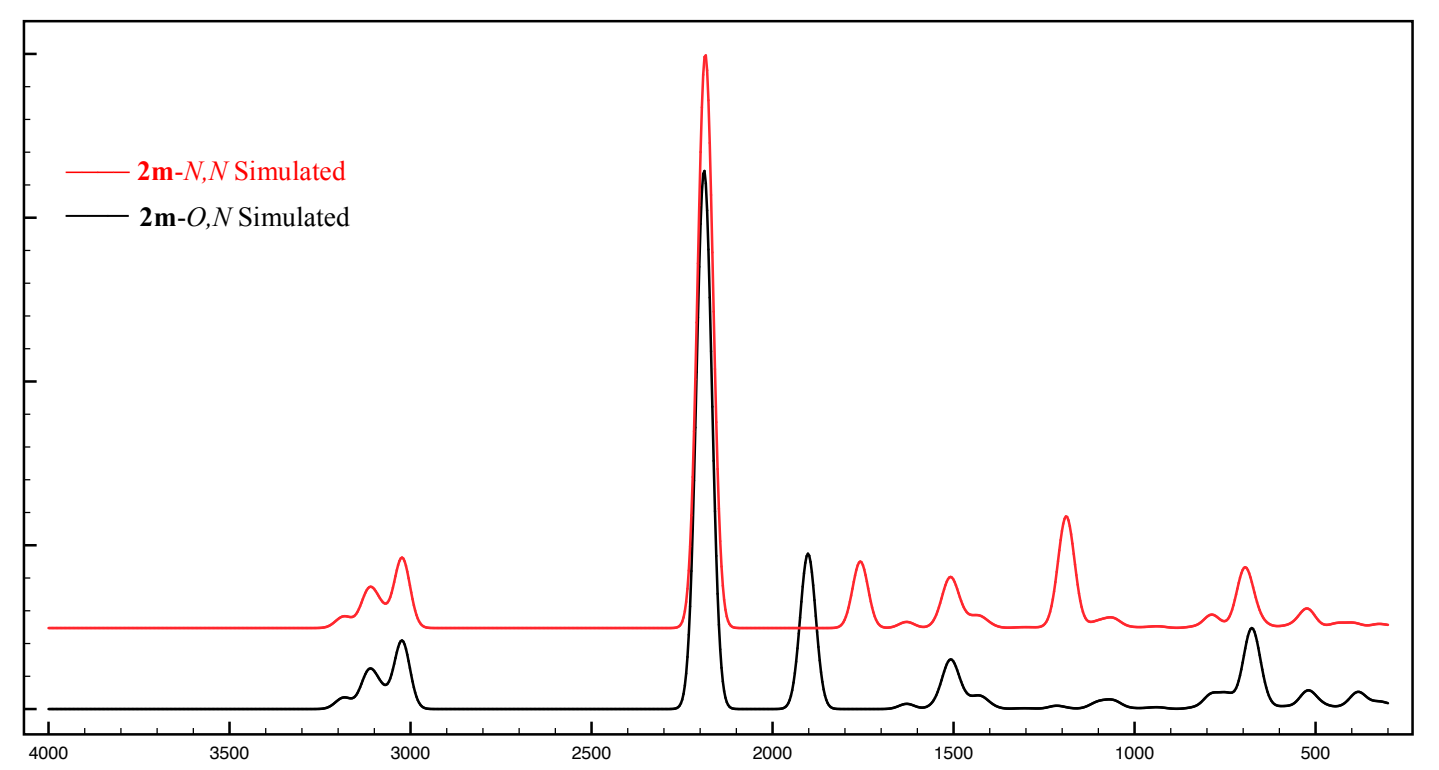

Figure S2.1. DFT-Simulated IR absorbance spectra of Cp* $\mathrm{Co}\left(\eta^{2}-N, N-\mathrm{N}_{2} \mathrm{O}\right)(\mathrm{CNXyl})(\mathbf{2 m}$ $N, N$; red), and $\mathrm{Cp}^{*} \mathrm{Co}\left(\eta^{2}-O, N-\mathrm{N}_{2} \mathrm{O}\right)(\mathrm{CNXyl})(\mathbf{2 m}-O, N$; black). All IR spectra have been scaled by $0.9671 .{ }^{8}$

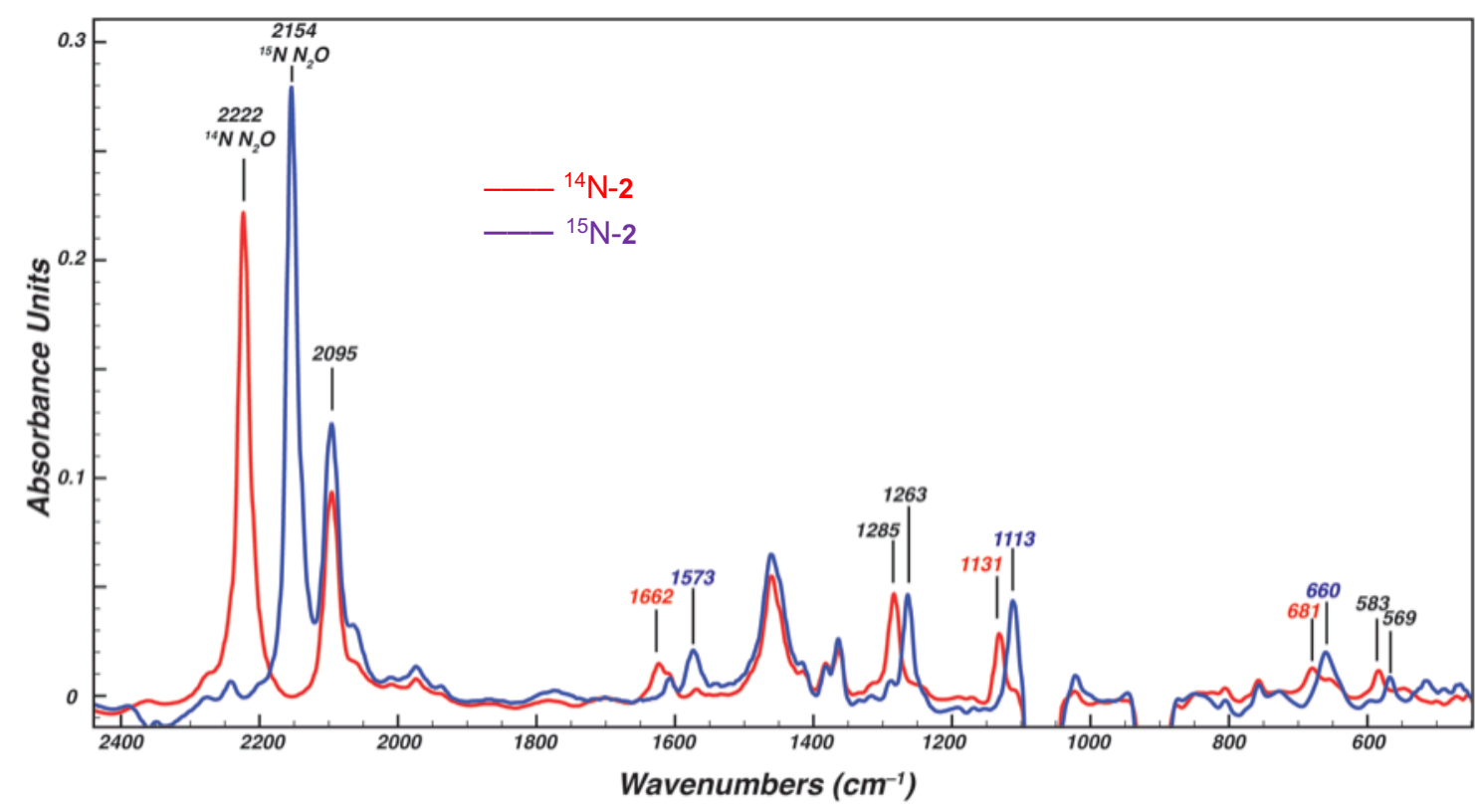

Figure S2.2. Full baseline corrected absorbance FTIR spectra in THF upon immediately warming to room temperature of $\mathrm{Cp} * \mathrm{Co}\left(\eta^{2}-\left({ }^{14} N,{ }^{14} N\right)-\mathrm{N}_{2} \mathrm{O}\right) \mathrm{CNAr}{ }^{\text {Tripp2 }}$ (Red) and $\mathrm{Cp} * \mathrm{Co}\left(\eta^{2}-\left({ }^{15} N,{ }^{15} N\right)-\mathrm{N}_{2} \mathrm{O}\right) \mathrm{CNAr}{ }^{\text {Tripp} 2}$ (Blue) denoting isotope sensitive vibrations and bends of the $\mathrm{N}_{2} \mathrm{O}$ ligand. Peaks denoted in black correspond to uncoordinated $\mathrm{N}_{2} \mathrm{O}$ gas. 


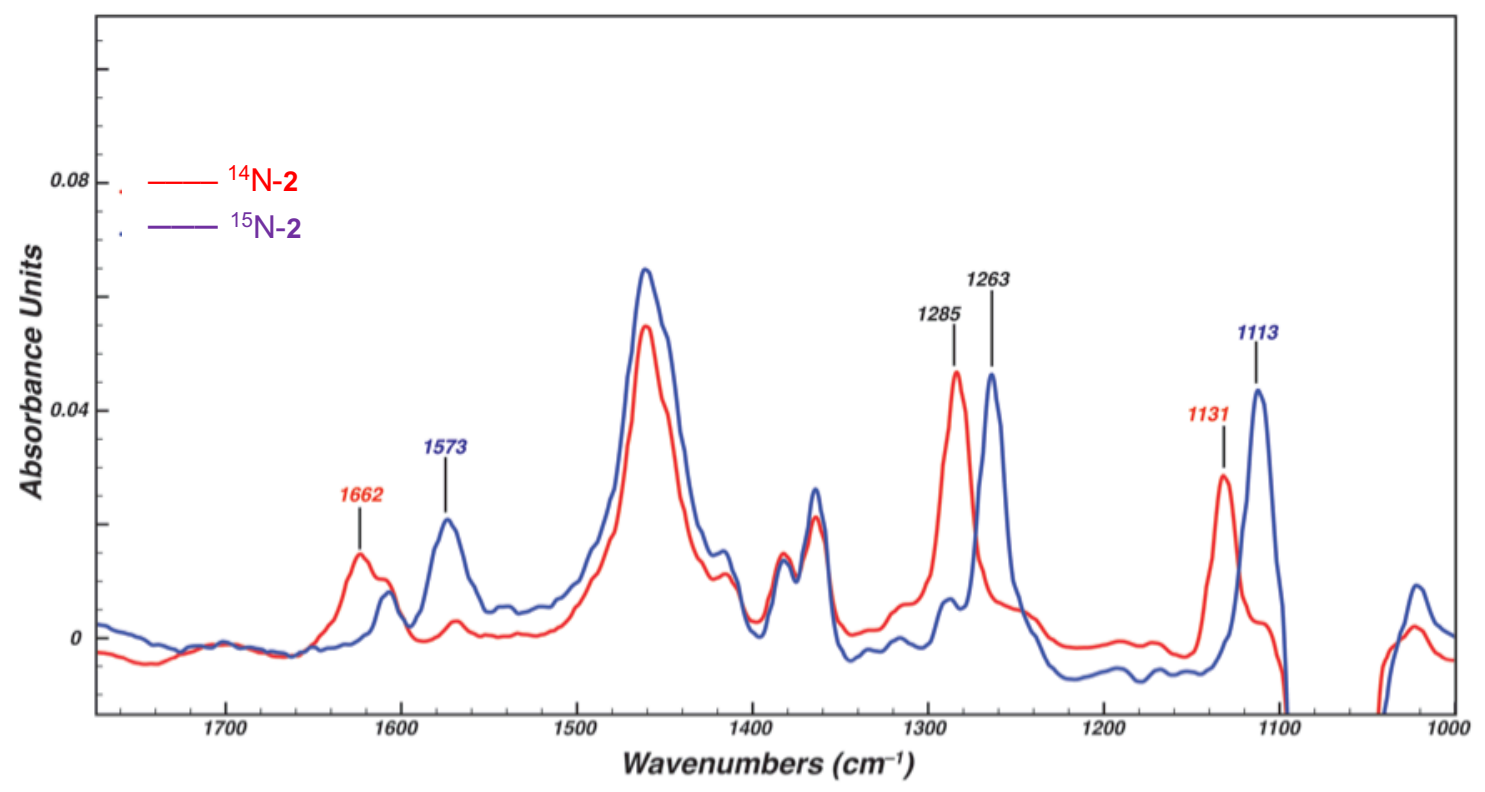

Figure S2.3. Zoomed in baseline corrected absorbance FTIR spectra $\left(1800-1000 \mathrm{~cm}^{-1}\right.$ region) in THF immediately warming to room temperature of $\mathrm{Cp} * \operatorname{Co}\left(\eta^{2}-\left({ }^{14} N,{ }^{14} \mathrm{~N}\right)\right.$ $\left.\mathrm{N}_{2} \mathrm{O}\right) \mathrm{CNAr}^{\text {Tripp2 }}$ (Red) and $\mathrm{Cp}^{*} \mathrm{Co}\left(\eta^{2}-\left({ }^{15} N{ }^{15}{ }^{15}\right)_{-}-\mathrm{N}_{2} \mathrm{O}\right) \mathrm{CNAr}^{\text {Tripp2 }}$ (Blue) denoting isotope sensitive vibrations and bends of the $\mathrm{N}_{2} \mathrm{O}$ ligand. Peaks denoted in black correspond to uncoordinated $\mathrm{N}_{2} \mathrm{O}$ gas.

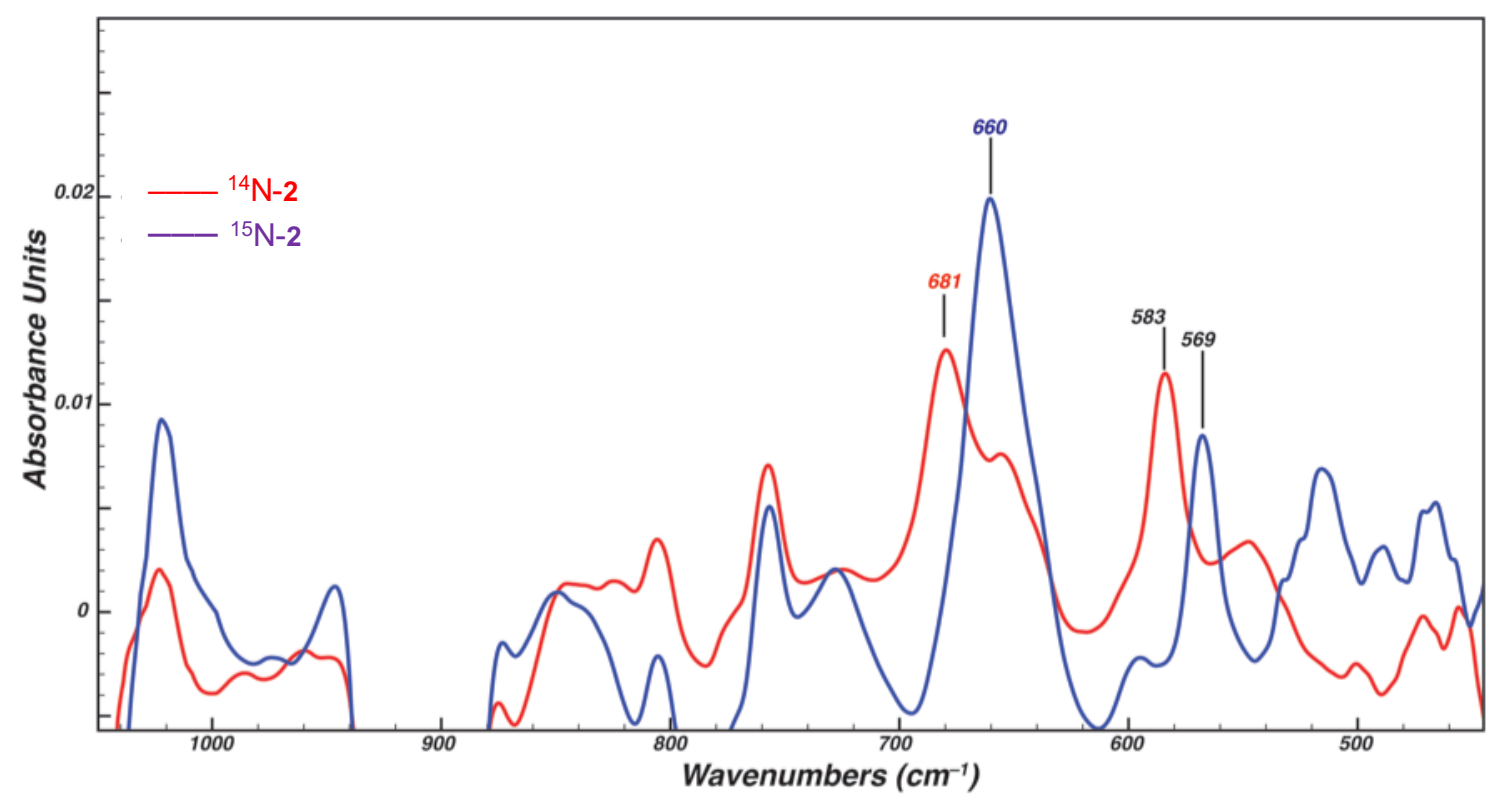

Figure S2.4. Zoomed in baseline corrected absorbance FTIR spectra $\left(1100-400 \mathrm{~cm}^{-1}\right.$ region) in THF immediately warming to room temperature of $\mathrm{Cp} * \operatorname{Co}\left(\eta^{2}-\left({ }^{14} N,{ }^{14} \mathrm{~N}\right)\right.$ $\left.\mathrm{N}_{2} \mathrm{O}\right) \mathrm{CNAr}^{\text {Tripp2 }}$ (Red) and $\mathrm{Cp} * \mathrm{Co}\left(\eta^{2}-\left({ }^{15} N,{ }^{15} N\right)-\mathrm{N}_{2} \mathrm{O}\right) \mathrm{CNAr}^{\text {Tripp2}}$ (Blue) denoting isotope sensitive vibrations and bends of the $\mathrm{N}_{2} \mathrm{O}$. Peaks denoted in black correspond to uncoordinated $\mathrm{N}_{2} \mathrm{O}$ gas. 


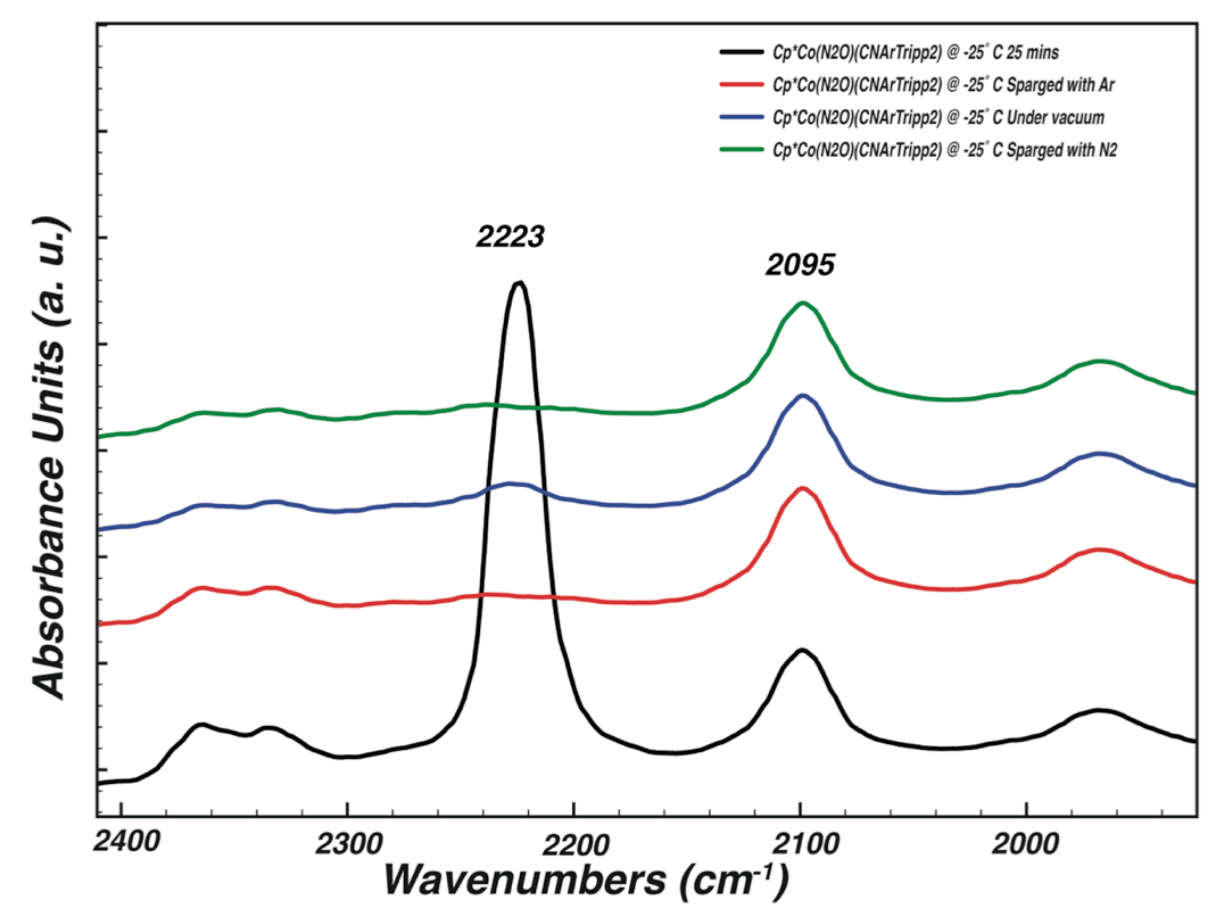

Figure S2.5. In situ solution FTIR spectra (THF, $-25^{\circ} \mathrm{C}$, isocyanide $v \mathrm{CN}$ region) depicting the generation of $\mathrm{Cp} * \mathrm{Co}\left(\eta^{2}-N, N-\mathrm{N}_{2} \mathrm{O}\right) \mathrm{CNAr}{ }^{\text {Tripp2 }}$ (black), and the stability under an $\operatorname{Ar}_{(\mathrm{g})}$ atmosphere (red), post exposure to vacuum (blue), and exposure to a $\mathrm{N}_{2(\mathrm{~g})}$ atmosphere (green). The band at $2223 \mathrm{~cm}^{-1}$ is from free $\mathrm{N}_{2} \mathrm{O}$.

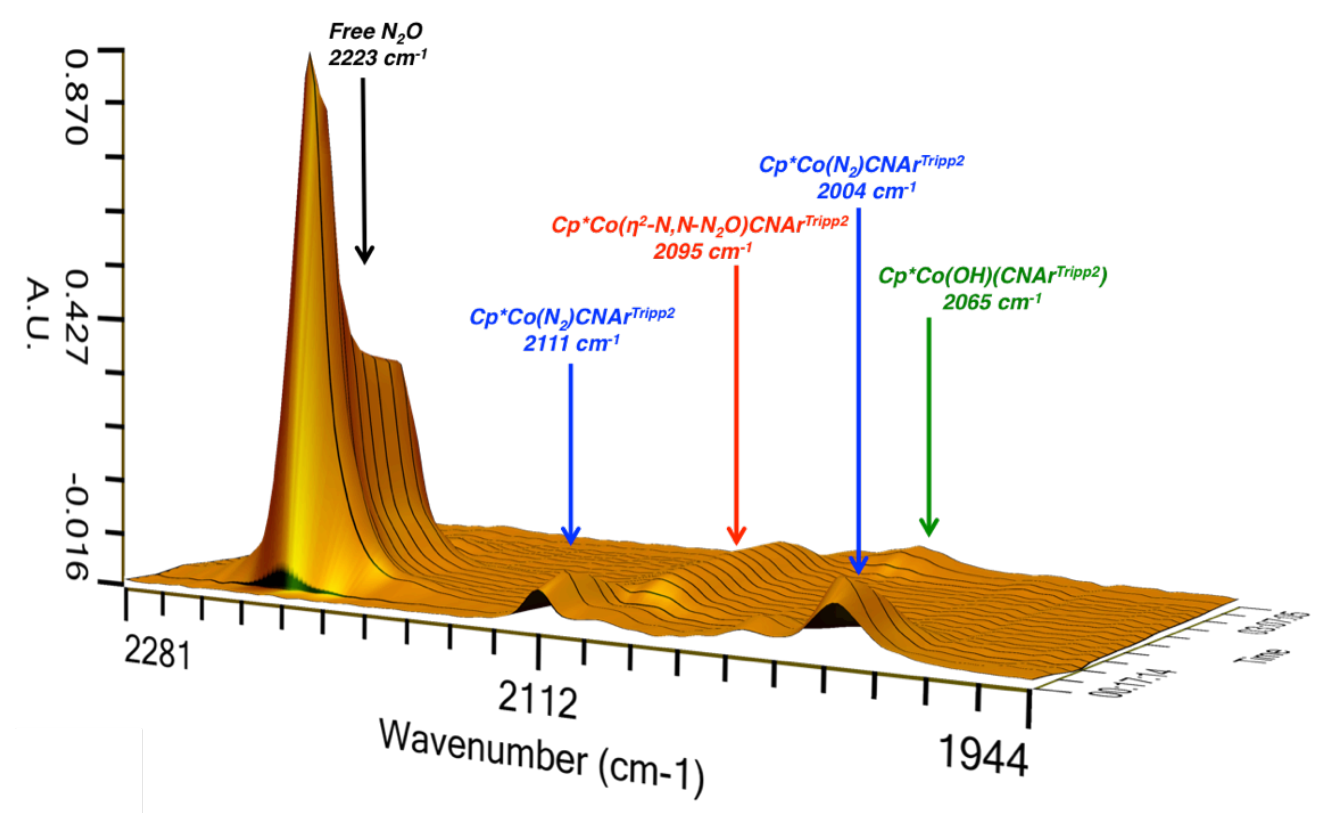

Figure S2.6. React-IR FTIR spectra v. Time (THF, $\left.-25{ }^{\circ} \mathrm{C}-22{ }^{\circ} \mathrm{C}\right)$ depicting the in situ formation of $\mathrm{Cp} * \mathrm{Co}\left(\eta^{2}-N, N-\mathrm{N}_{2} \mathrm{O}\right) \mathrm{CNAr}{ }^{\text {Tripp2 }}$ and subsequent decomposition to $\mathrm{Cp}^{*} \mathrm{Co}(\mathrm{OH})\left(\mathrm{CNAr}^{\mathrm{Tripp} 2}\right)$ upon sparging with $\mathrm{Ar}_{(\mathrm{g})}$ and warming to room temperature. 


\section{S3. Selected NMR Spectra}

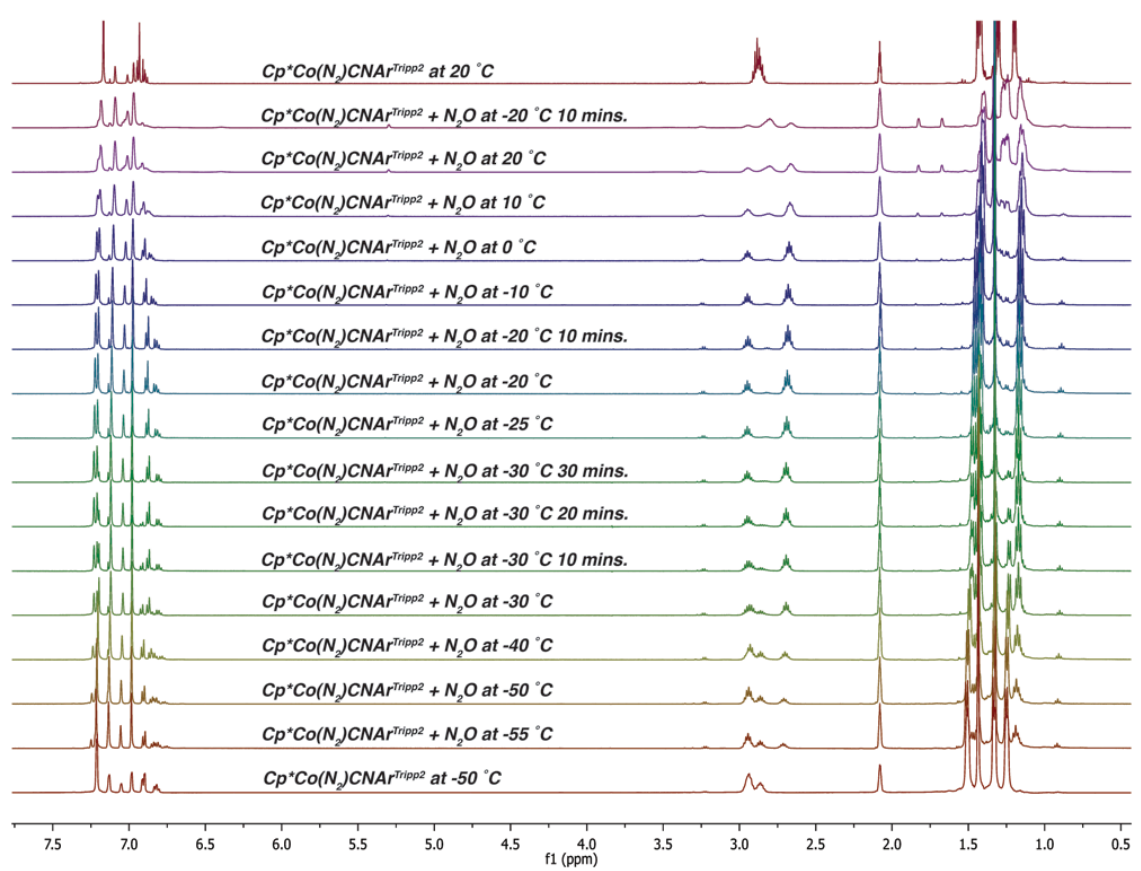

Figure S3.1. Full variable temperature ${ }^{1} \mathrm{H}$ NMR spectra $\left(499.8 \mathrm{MHz}\right.$, Tol- $\left.d^{8}\right)$ of the generation of $\mathrm{Cp} * \mathrm{Co}\left(\eta^{2}-N, N-\mathrm{N}_{2} \mathrm{O}\right) \mathrm{CNAr}{ }^{\text {Tripp2 }}$. Note the top, and bottom spectrum depicts the starting material $\left(\mathrm{Cp}^{*} \mathrm{Co}\left(\mathrm{N}_{2}\right) \mathrm{CNAr}{ }^{\text {Tripp} 2}\right)$ in Tol- $d^{8}$ at $20{ }^{\circ} \mathrm{C}$, and $-55^{\circ} \mathrm{C}$ respectfully.

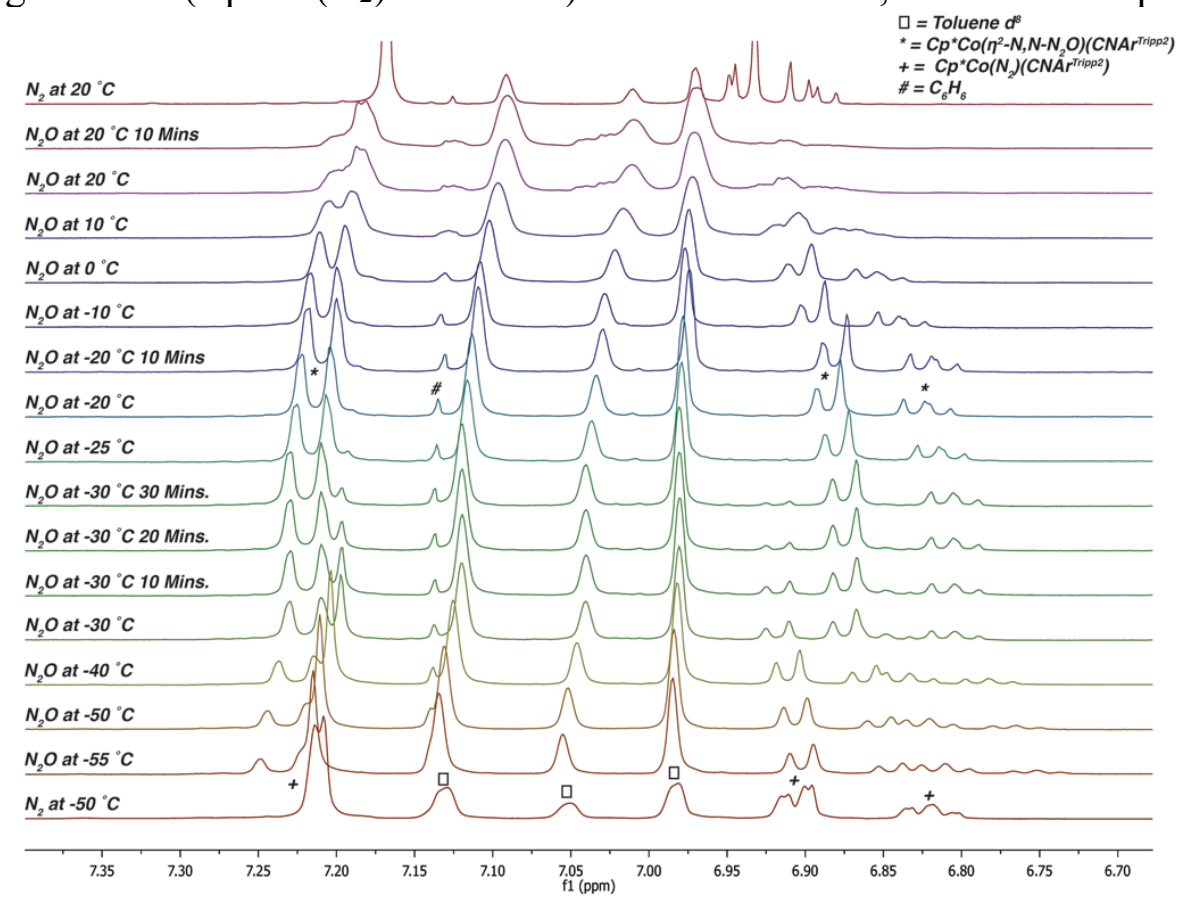

Figure S3.2. Zoomed in variable temperature ${ }^{1} \mathrm{H}$ NMR spectra (499.8 MHz, Tol- $d^{8}$ ) of the generation of $\mathrm{Cp}^{*} \mathrm{Co}\left(\eta^{2}-N, N-\mathrm{N}_{2} \mathrm{O}\right) \mathrm{CNAr}{ }^{\text {Tripp2 }}$ focused at the aromatic region. Note the top, and bottom spectrum depicts the starting material $\left(\mathrm{Cp}^{*} \mathrm{Co}\left(\mathrm{N}_{2}\right) \mathrm{CNAr}^{T r i p p} 2\right)$ in at $20{ }^{\circ} \mathrm{C}$, and $-55{ }^{\circ} \mathrm{C}$ respectfully. 


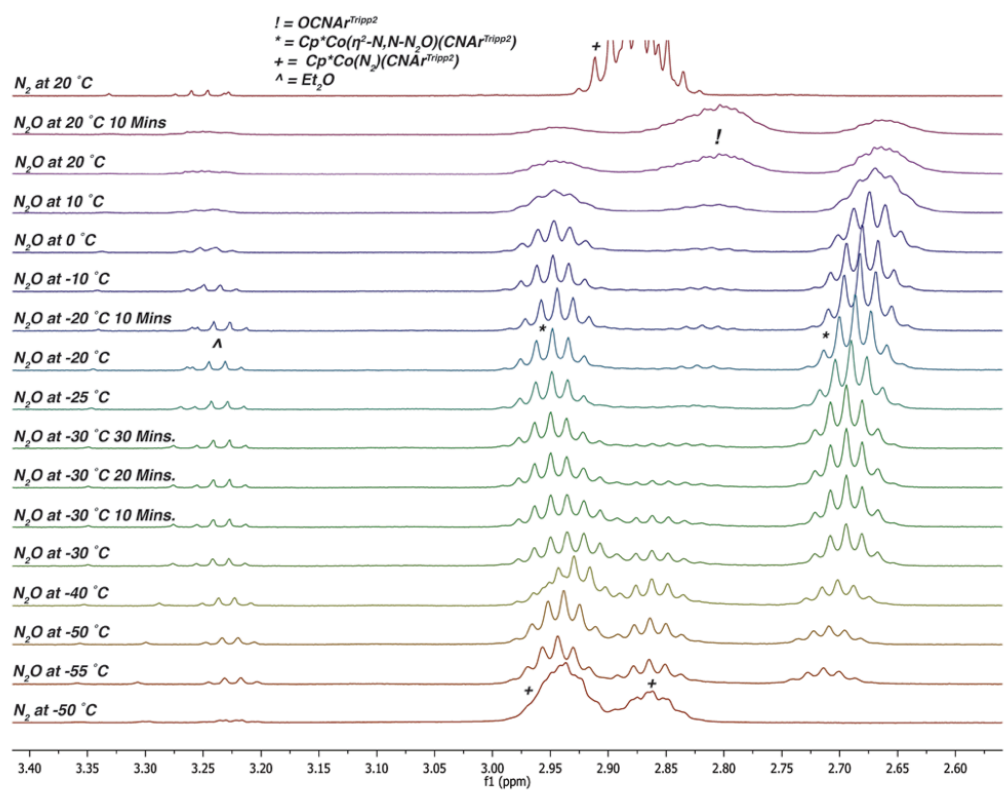

Figure S3.3 Zoomed in variable temperature ${ }^{1} \mathrm{H}$ NMR spectra $\left(499.8 \mathrm{MHz}\right.$, Tol- $\left.d^{8}\right)$ of the generation of $\mathrm{Cp} * \mathrm{Co}\left(\eta^{2}-N, N-\mathrm{N}_{2} \mathrm{O}\right) \mathrm{CNAr}{ }^{\text {Tripp2 }}$ focused at the isopropyl methylene proton region $\left(\mathrm{CH}\left(\mathrm{CH}_{3}\right)_{2}\right)$. Note the top, and bottom spectrum depicts the starting material $\left(\mathrm{Cp}^{*} \mathrm{Co}\left(\mathrm{N}_{2}\right) \mathrm{CNAr}{ }^{\text {Tripp} 2}\right)$ in Tol- $d^{8}$ at $20{ }^{\circ} \mathrm{C}$, and $-55{ }^{\circ} \mathrm{C}$ respectfully. At $\sim 10{ }^{\circ} \mathrm{C}$ the observation of a new septet at $2.80 \mathrm{ppm}$ with is attributed to OCNAr ${ }^{\text {Tripp2 }}$.

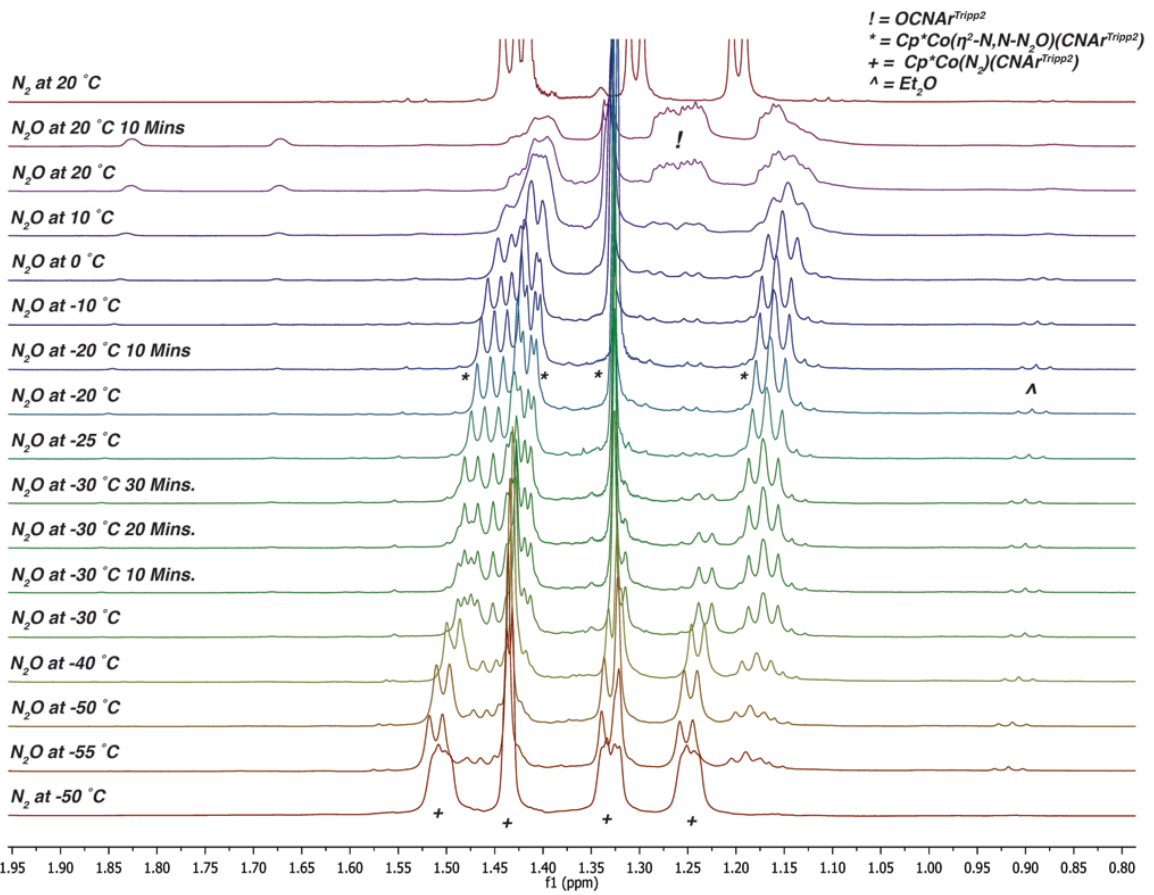

Figure S3.4. Zoomed in variable temperature ${ }^{1} \mathrm{H}$ NMR spectra (499.8 MHz, Tol- $d^{8}$ ) spectrum of the generation of $\mathrm{Cp}^{*} \mathrm{Co}\left(\eta^{2}-N, N-\mathrm{N}_{2} \mathrm{O}\right) \mathrm{CNAr}{ }^{\text {Tripp2 }}$ focused at the aliphatic proton region $\left(\mathrm{CH}\left(\mathrm{CH}_{3}\right)_{2}\right)$. Note the top, and bottom spectrum depicts the starting material $\left(\mathrm{Cp}^{*} \mathrm{Co}\left(\mathrm{N}_{2}\right) \mathrm{CNAr}{ }^{\text {Tripp} 2}\right)$ at $20{ }^{\circ} \mathrm{C}$, and $-55^{\circ} \mathrm{C}$ respectfully. 


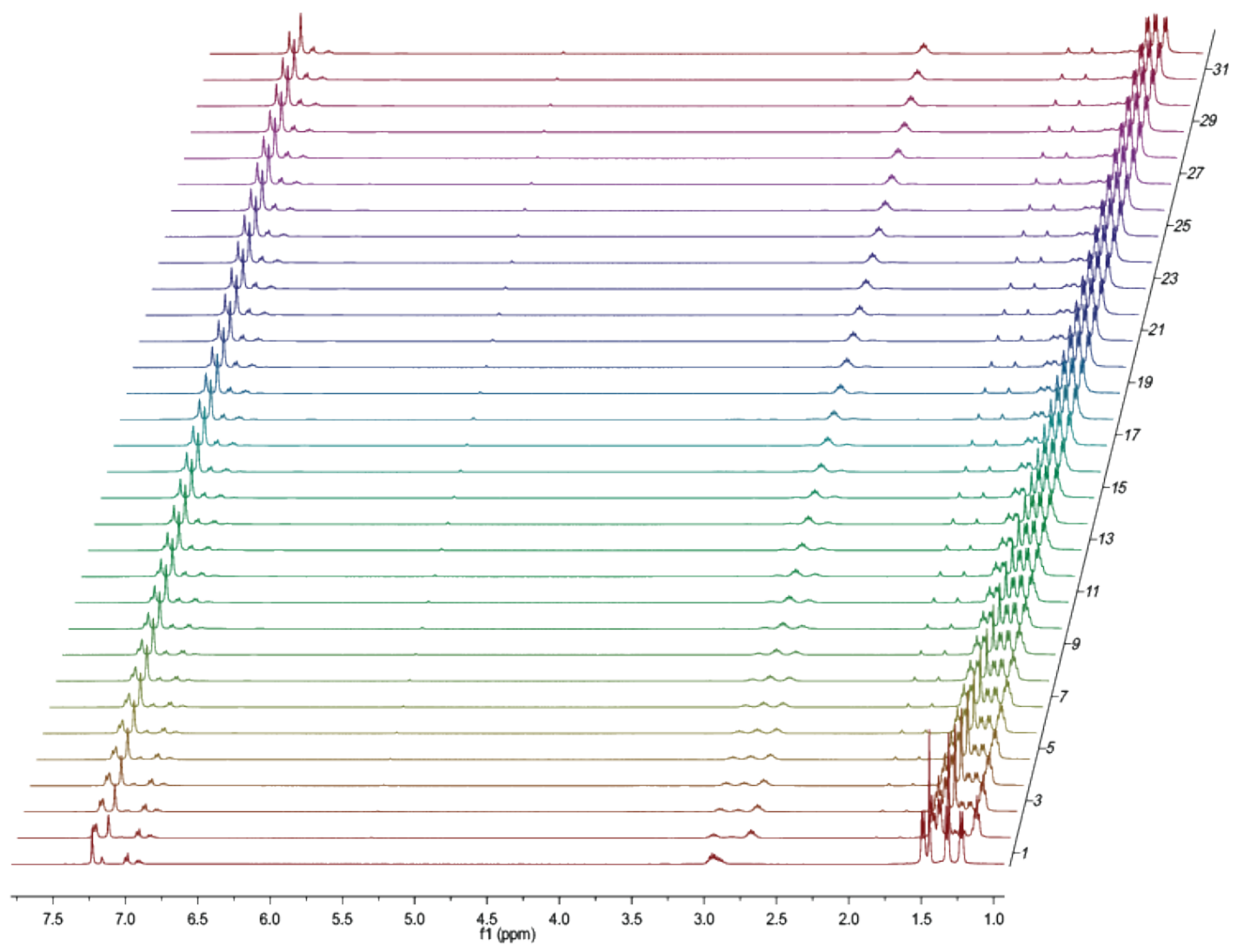

Figure S3.5. Full ${ }^{1} \mathrm{H}$ NMR spectra $\left(399.9 \mathrm{MHz}, \mathrm{C}_{6} \mathrm{D}_{6}\right)$ of the generation of $\mathrm{Cp} * \mathrm{Co}\left(\eta^{2}-\right.$ $\left.N, N-\mathrm{N}_{2} \mathrm{O}\right) \mathrm{CNAr}{ }^{\text {Tripp2 }}$ and subsequent decomposition. The bottom spectrum depicts the starting material $\left(\mathrm{Cp}^{*} \mathrm{Co}\left(\mathrm{N}_{2}\right) \mathrm{CNAr}{ }^{\text {Tripp} 2}\right)$ in $\mathrm{C}_{6} \mathrm{D}_{6}$ at $22{ }^{\circ} \mathrm{C}$. 


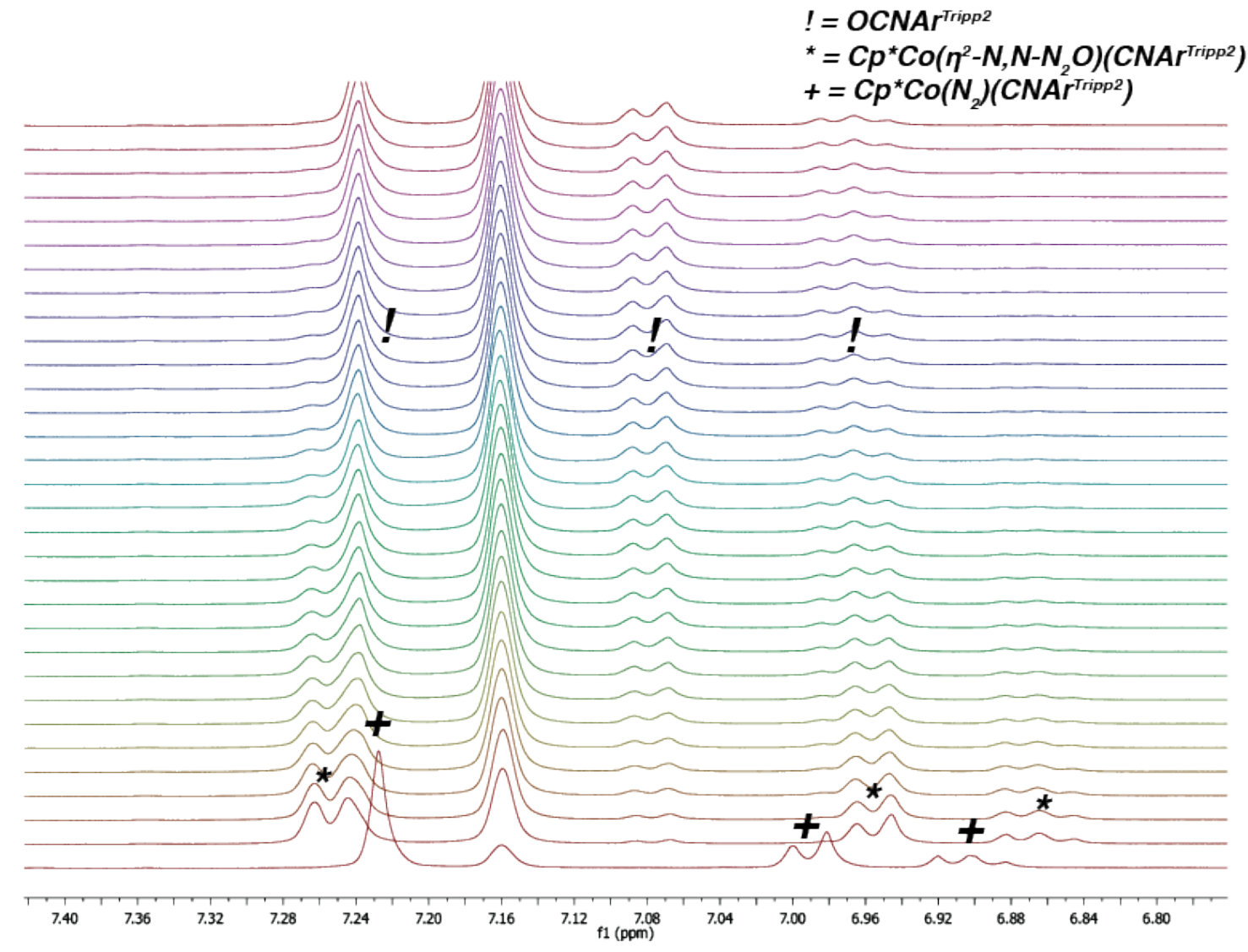

Figure S3.6. Zoomed in room temperature ${ }^{1} \mathrm{H}$ NMR spectra $\left(399.9 \mathrm{MHz}, \mathrm{C}_{6} \mathrm{D}_{6}\right)$ of the generation of $\mathrm{Cp} * \mathrm{Co}\left(\eta^{2}-N, N-\mathrm{N}_{2} \mathrm{O}\right) \mathrm{CNAr}{ }^{\text {Tripp2 }}$ and subsequent decomposition to OCNAr ${ }^{\text {Tripp } 2}$ focused at the aromatic region. Note the bottom spectrum depicts the starting material $\left(\mathrm{Cp}^{*} \mathrm{Co}\left(\mathrm{N}_{2}\right) \mathrm{CNAr}^{\text {Tripp} 2}\right)$ in at $22{ }^{\circ} \mathrm{C}$. 


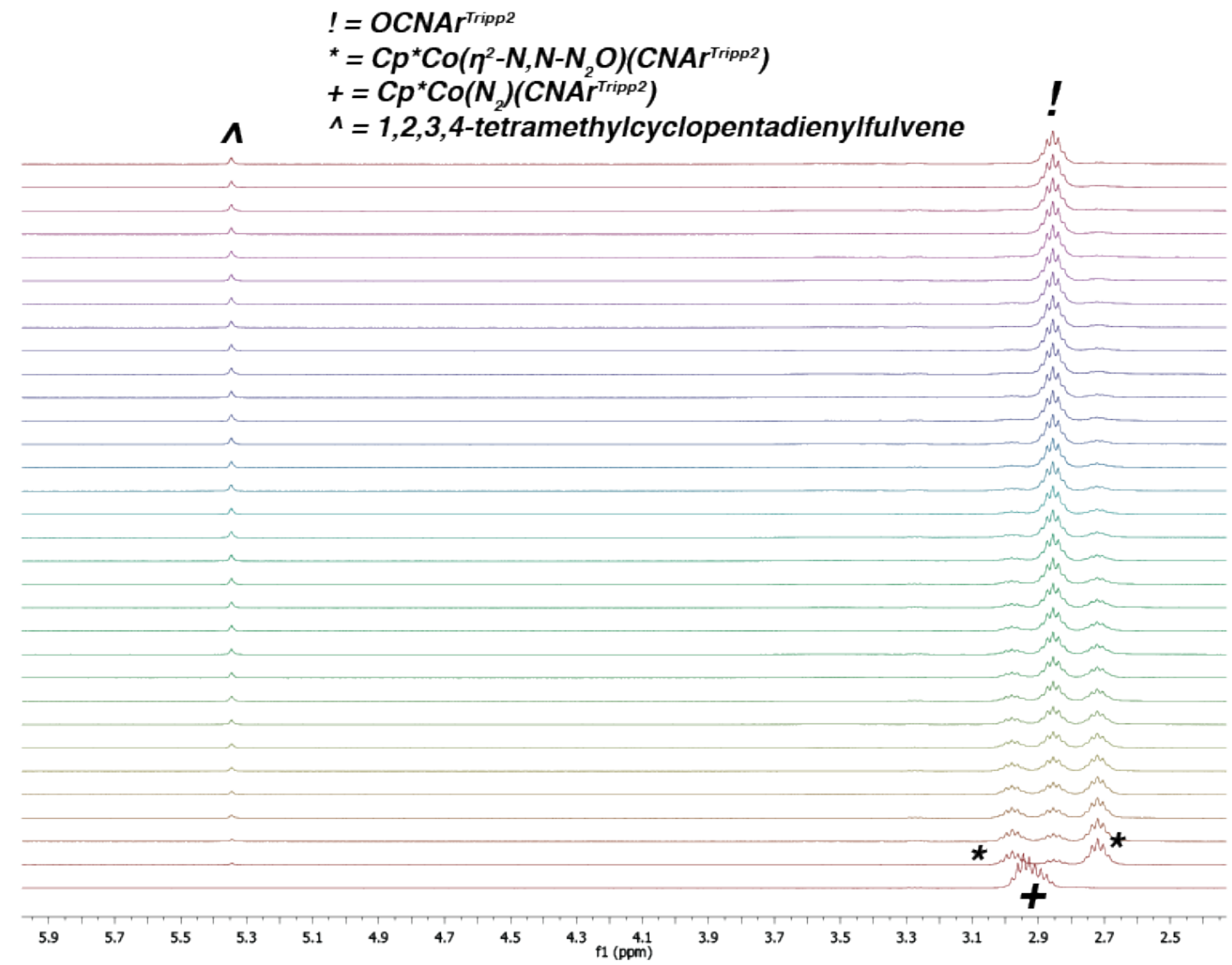

Figure S3.7. Zoomed in room temperature ${ }^{1} \mathrm{H}$ NMR spectra $\left(399.9 \mathrm{MHz}, \mathrm{C}_{6} \mathrm{D}_{6}\right)$ of the generation of $\mathrm{Cp} * \mathrm{Co}\left(\eta^{2}-N, N-\mathrm{N}_{2} \mathrm{O}\right) \mathrm{CNAr}{ }^{\text {Tripp2 }}$ and subsequent decomposition to OCNAr ${ }^{\text {Tripp2 }}$ focused at 2.0-5.9 ppm. Note the bottom spectrum depicts the starting material $\left(\mathrm{Cp}^{*} \mathrm{Co}\left(\mathrm{N}_{2}\right) \mathrm{CNAr}{ }^{\text {Tripp2 }}\right)$ at $22{ }^{\circ} \mathrm{C}$. The singlet at $5.31 \mathrm{ppm}$ indicates formation of 1,2,3,4-tetramenthylcyclopentadienylfulvene. ${ }^{9}$ 


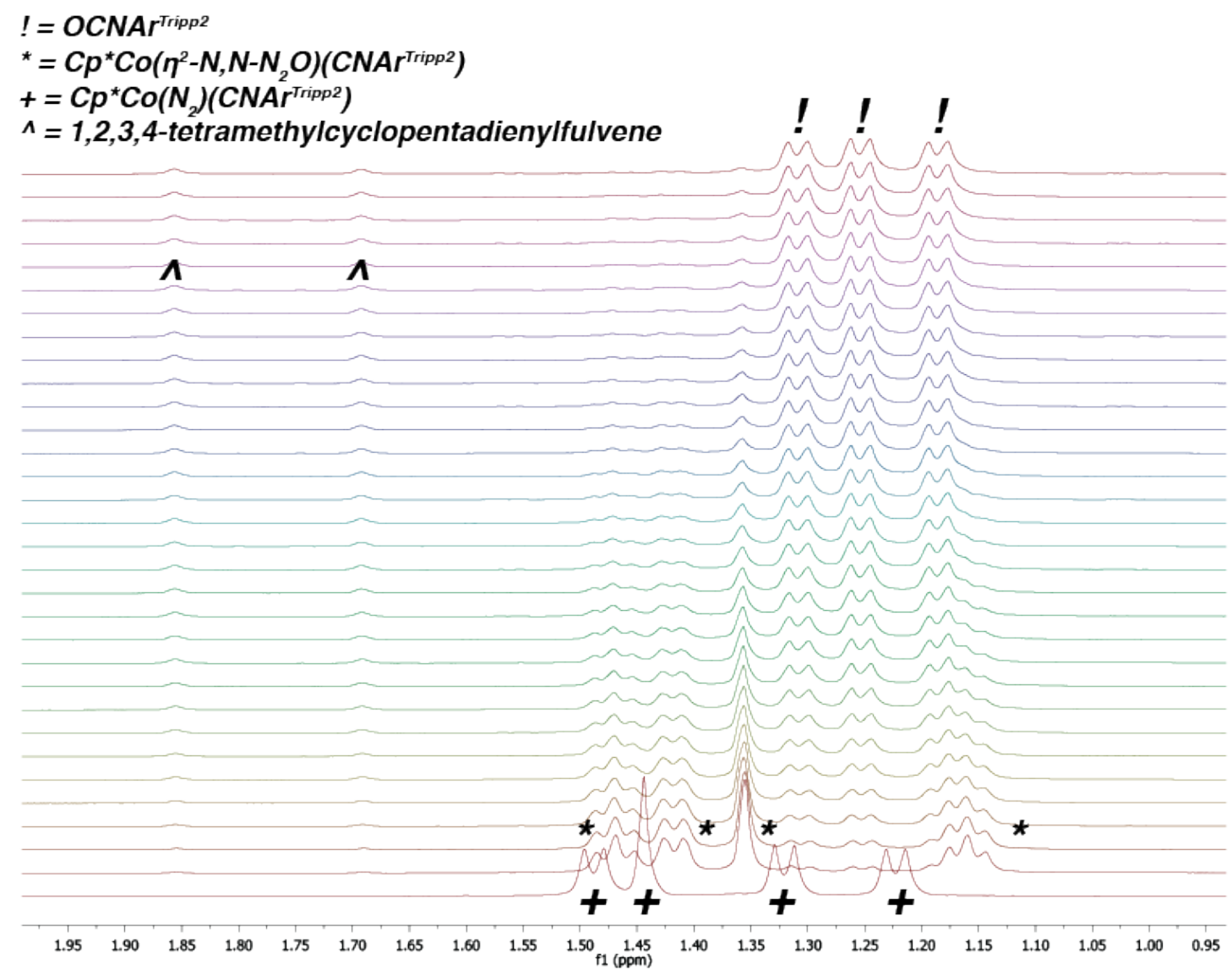

Figure S3.8. Zoomed in room temperature ${ }^{1} \mathrm{H}$ NMR spectra $\left(399.9 \mathrm{MHz}, \mathrm{C}_{6} \mathrm{D}_{6}\right)$ of the generation of $\mathrm{Cp} * \mathrm{Co}\left(\eta^{2}-N, N-\mathrm{N}_{2} \mathrm{O}\right) \mathrm{CNAr}{ }^{\text {Tripp2 }}$ and subsequent decomposition to OCNAr ${ }^{\text {Tripp2 }}$ focused at 0.95-1.95 ppm. Note the bottom spectrum depicts the starting material $\left(\mathrm{Cp}^{*} \mathrm{Co}\left(\mathrm{N}_{2}\right) \mathrm{CNAr}^{\text {Tripp}} 2\right)$ at $22{ }^{\circ} \mathrm{C}$. The singlet at 1.70 and $1.85 \mathrm{ppm}$ indicates formation of 1,2,3,4-tetramenthylcyclopentadienylfulvene. ${ }^{9}$ 


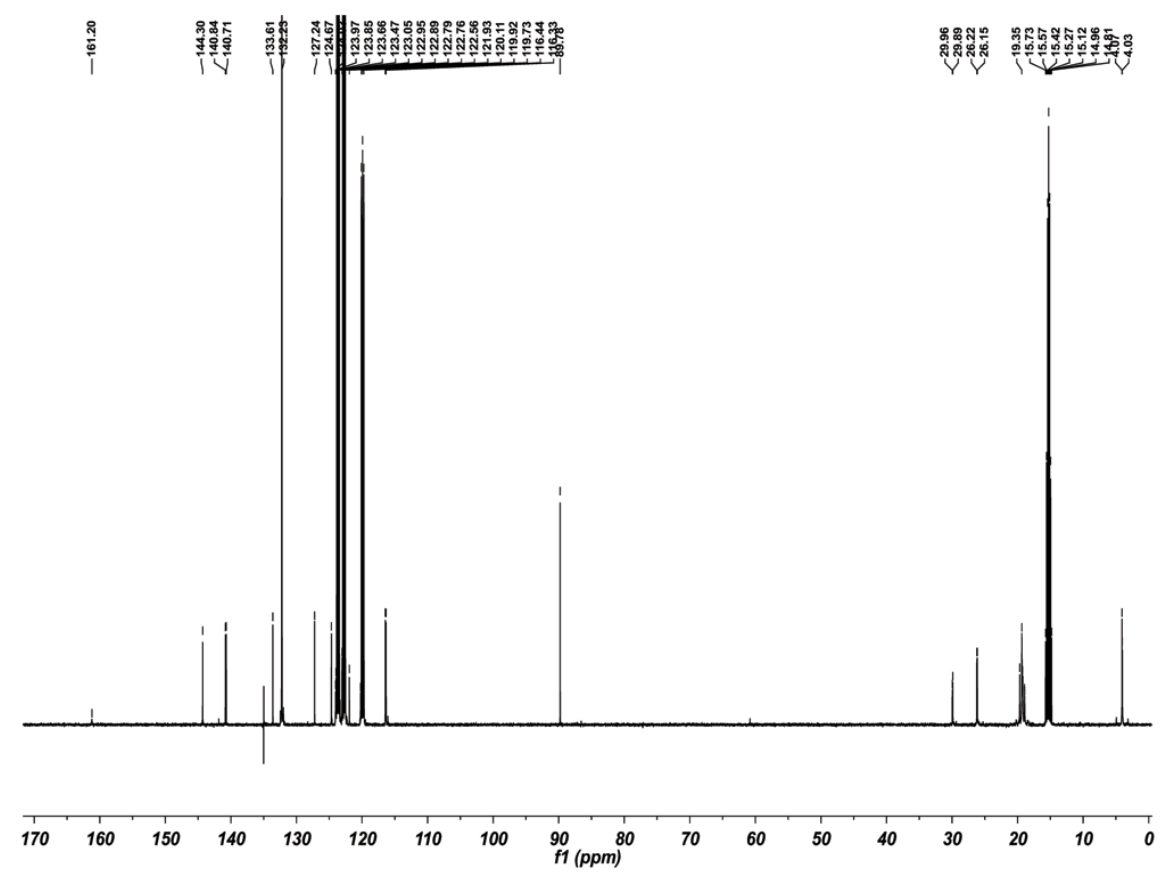

Figure S3.9. ${ }^{13} \mathrm{C}\left\{{ }^{1} \mathrm{H}\right\}$ NMR spectrum $\left(\mathrm{Tol}-d 8,125.8 \mathrm{MHz},-20{ }^{\circ} \mathrm{C}\right)$ of $\mathrm{Cp} * \mathrm{Co}\left(\eta^{2}-N, N-\right.$ $\left.\mathrm{N}_{2} \mathrm{O}\right) \mathrm{CNAr}^{\text {Tripp2}}$.

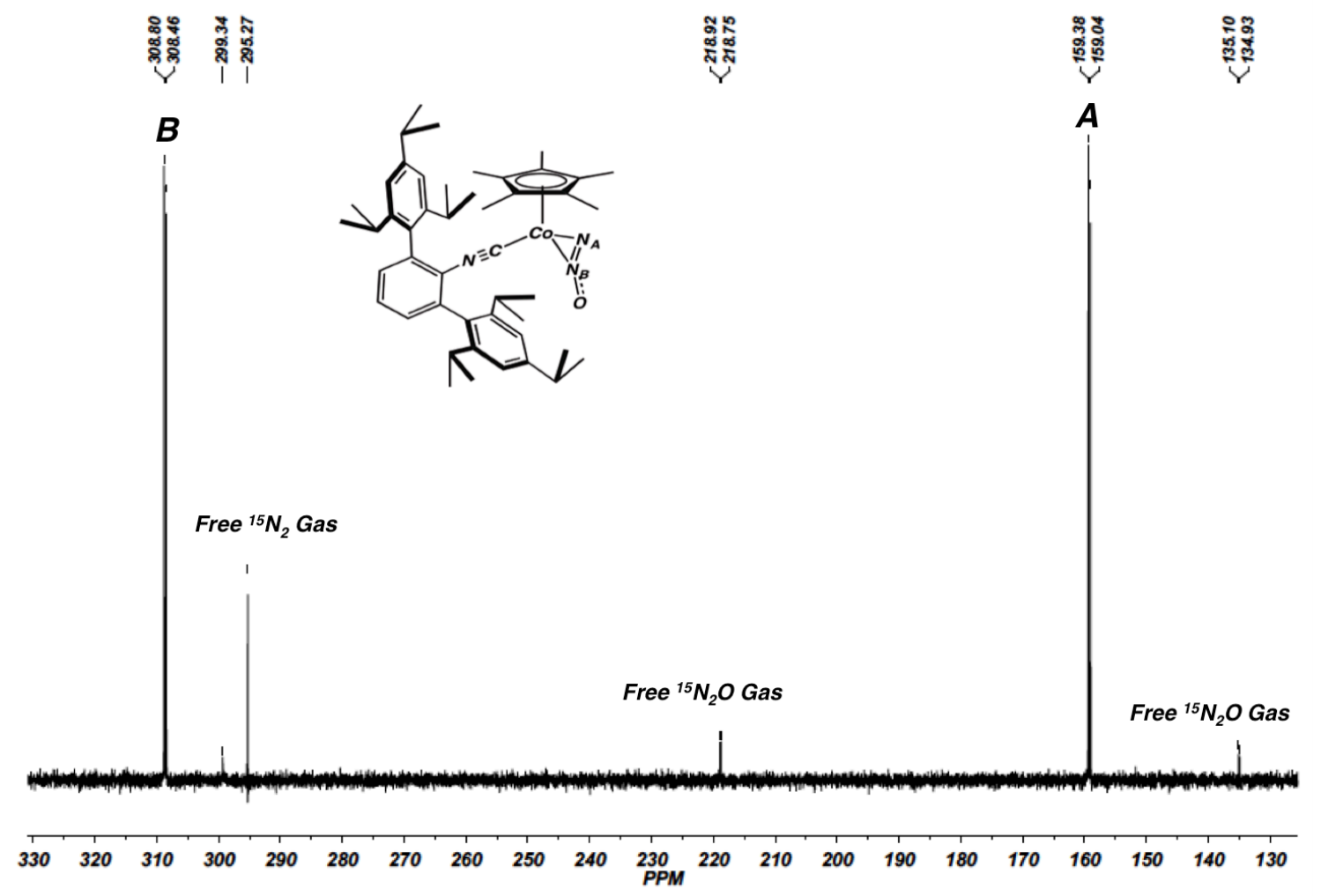

Figure S3.10. ${ }^{15} \mathrm{~N}\left\{{ }^{1} \mathrm{H}\right\}$ NMR spectrum $\left(\mathrm{Tol}-d^{8}, 50.7 \mathrm{MHz},-20{ }^{\circ} \mathrm{C}\right)$ of $\mathrm{Cp} * \mathrm{Co}\left(\eta^{2}-N, N-\right.$ $\left.{ }^{15} \mathrm{~N}_{2} \mathrm{O}\right) \mathrm{CNAr}{ }^{\text {Tripp} 2}$ referenced to $\mathrm{CH}_{3} \mathrm{NO}$. 


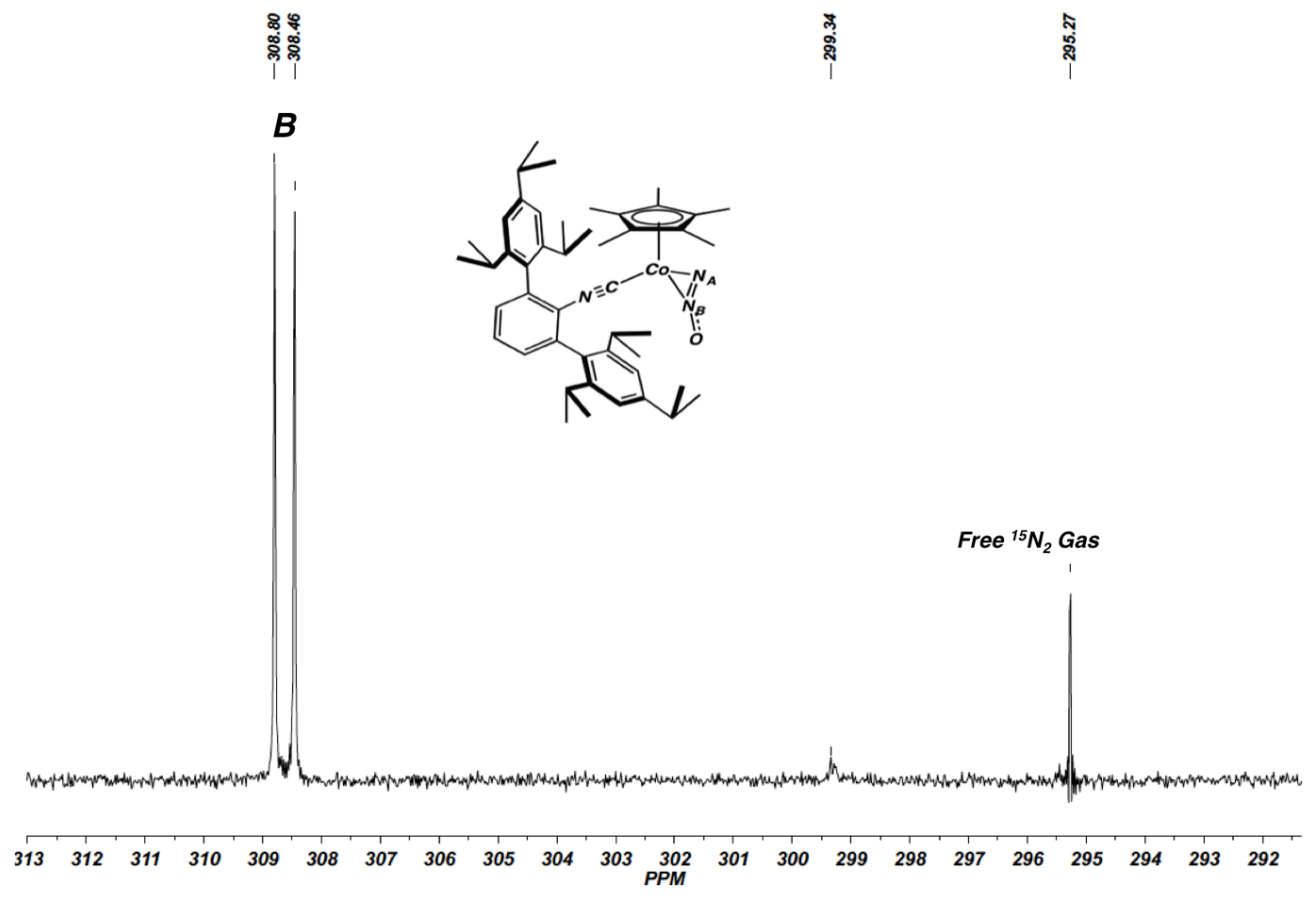

Figure S3.11. ${ }^{15} \mathrm{~N}\left\{{ }^{1} \mathrm{H}\right\}$ NMR spectrum (Tol- $d^{8}, 50.7 \mathrm{MHz},-20{ }^{\circ} \mathrm{C}$ ) zoom in of $\mathrm{Cp}{ }^{*} \mathrm{Co}\left(\eta^{2}-N, N_{-}{ }^{15} \mathrm{~N}_{2} \mathrm{O}\right) \mathrm{CNAr}^{\text {Tripp} 2}$ referenced to $\mathrm{CH}_{3} \mathrm{NO}$.

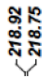

율ำ
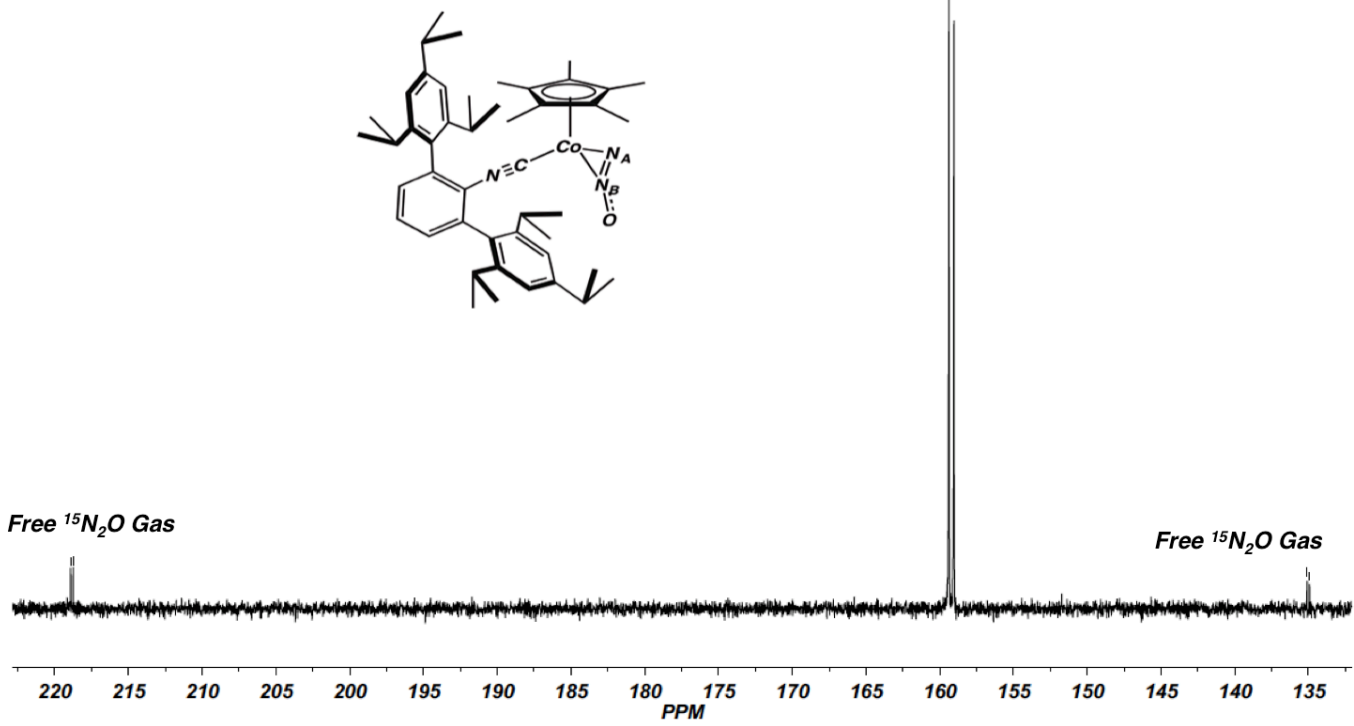

Figure S3.12. ${ }^{15} \mathrm{~N}\left\{{ }^{1} \mathrm{H}\right\}$ NMR spectrum (Tol- $\left.d^{8}, 50.7 \mathrm{MHz},-20{ }^{\circ} \mathrm{C}\right)$ zoom in of $\mathrm{Cp}{ }^{*} \mathrm{Co}\left(\eta^{2}-N, N_{-}{ }^{15} \mathrm{~N}_{2} \mathrm{O}\right) \mathrm{CNAr}^{\text {Tripp} 2}$ referenced to $\mathrm{CH}_{3} \mathrm{NO}$. 


\section{S4. Computational Results}

S4.1. Computational Details. Density Functional Theory (DFT) calculations were carried out on $\mathrm{Cp}^{*} \mathrm{Co}\left(\eta^{2}-N, N-\mathrm{N}_{2} \mathrm{O}\right)\left(\mathrm{CN}^{\mathrm{Xylyl}}\right)$, and $\mathrm{Cp}^{*} \mathrm{Co}\left(\eta^{2}-N, O-\mathrm{N}_{2} \mathrm{O}\right)\left(\mathrm{CN}^{\mathrm{Xylyl}}\right)$, which are truncated models of the structurally characterized complex $\mathrm{Cp} * \mathrm{Co}\left(\eta^{2}-N, N-\mathrm{N}_{2} \mathrm{O}\right) \mathrm{CNAr}^{\text {Tripp2 }}$. Calculations were carried out using the ORCA program package. ${ }^{10}$ Geometry optimizations and single-point calculations were performed using the B3LYP hybrid density functional. ${ }^{11-12}$ The all-electron Ahlrichs triple-zeta basis sets def2-TZVP (standard) $)^{13}$ and def2-TZVP/J (auxiliary) ${ }^{14}$ were used in all calculations. The resolution of identity (RI) approximation was employed. ${ }^{15}$ Relativistic effects were included by use of the zerothorder regular approximation (ZORA). ${ }^{16-19}$ Crystallographic atomic coordinates were used as input for geometry optimizations where appropriate. Simulated frequency calculations were conducted on geometry optimized coordinates and calibrated according to literature values. ${ }^{8}$ Viewing of optimized structures and rendering of molecular orbitals was performed using the program Chemcraft. ${ }^{20}$ After each geometry optimization, the optimized coordinates were reoriented so as to make the $\mathrm{Cp}^{*}$ ring coplanar with the Cartesian $x y$ plane. These coordinates were then used to run a single point calculation to de-convolute orbital contributions. This research was supported in part by the W. M. Keck Foundation through computing resources at the W. M. Keck Laboratory for Integrated Biology II.

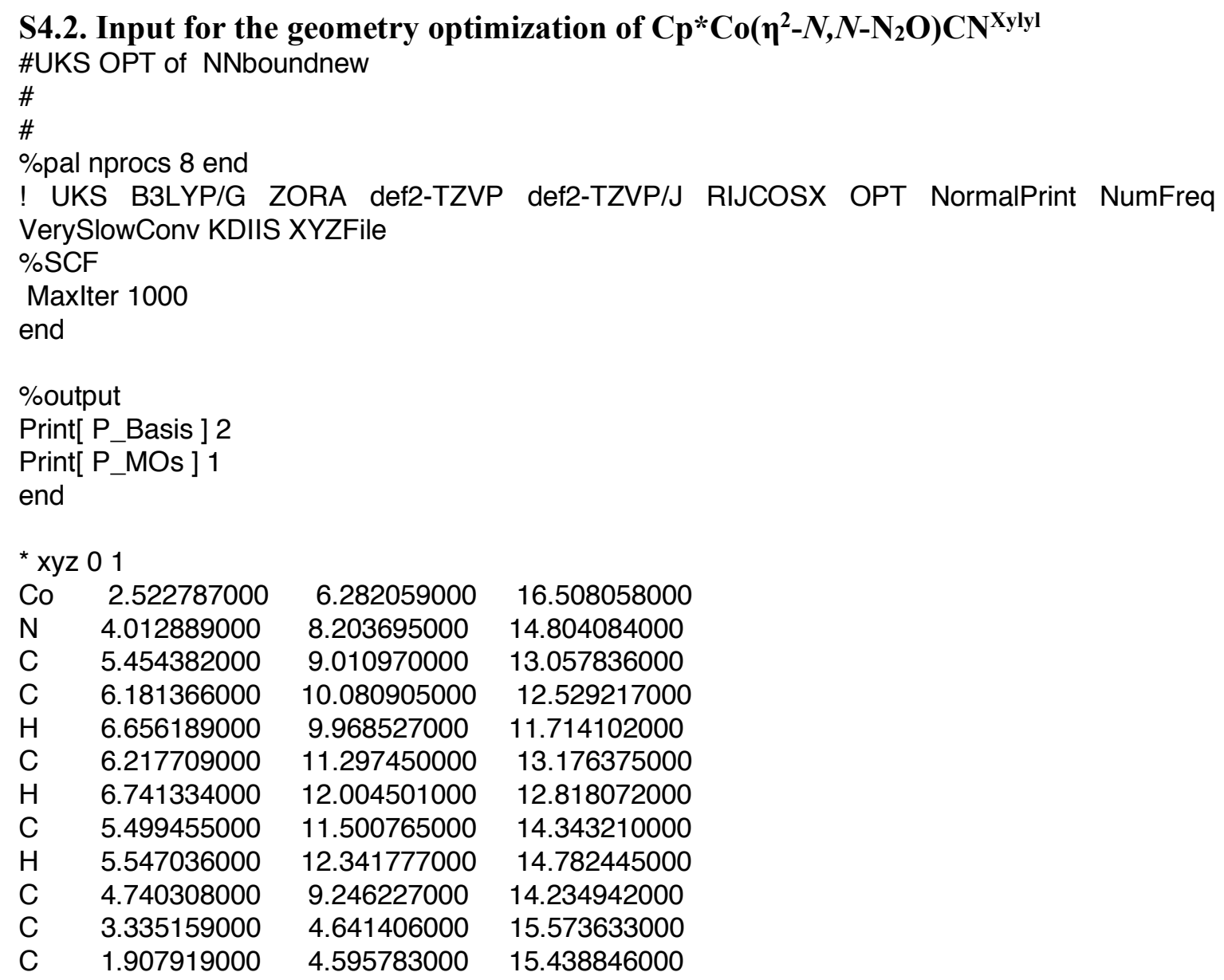




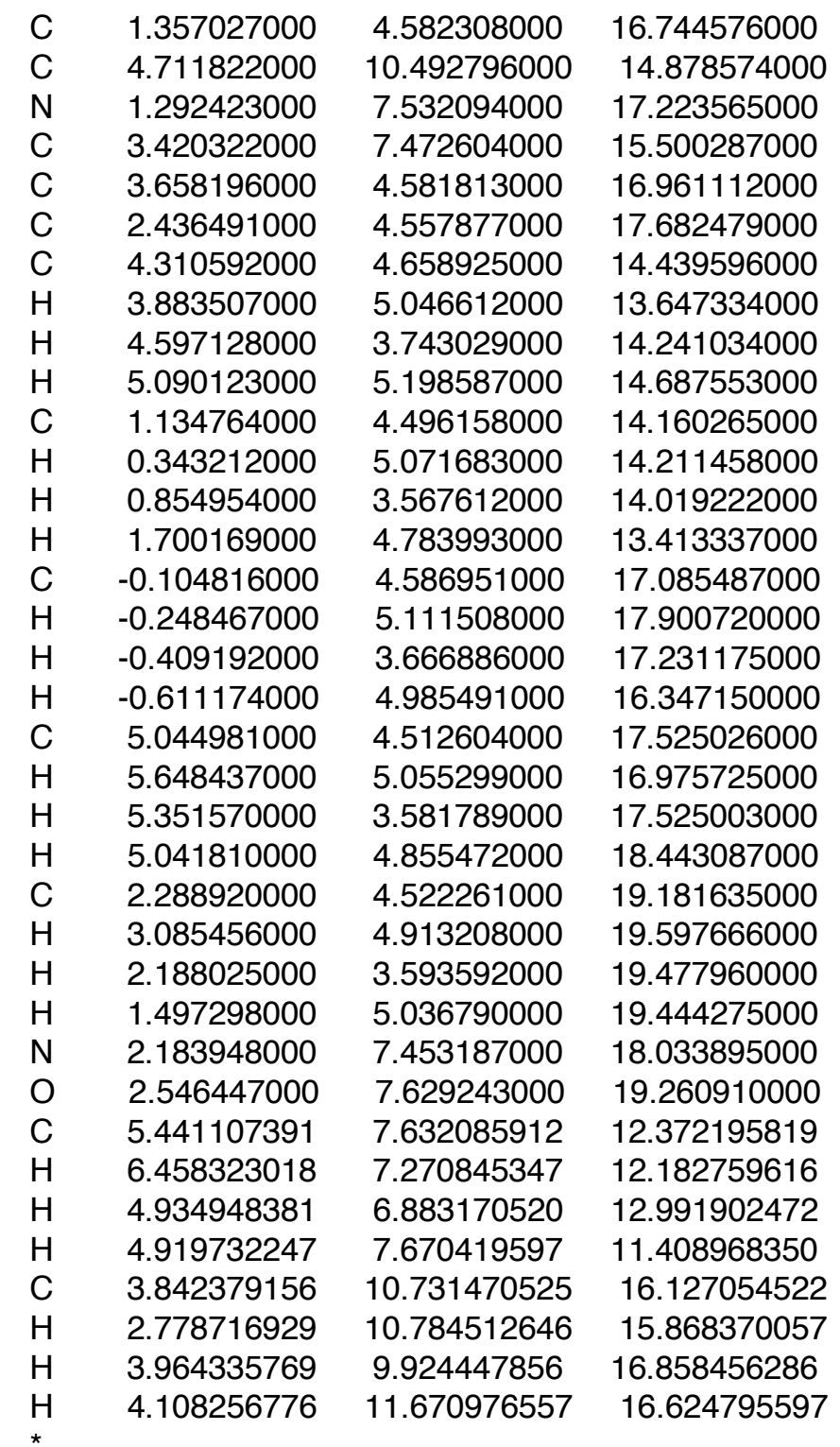

S4.3. Optimized coordinates for $\mathrm{Cp} * \mathrm{Co}\left(\eta^{2}-N, N-\mathrm{N}_{2} \mathrm{O}\right) \mathrm{CN}^{\mathrm{Xyly1}}$

$\begin{array}{cccc}\text { Co } & 2.75385717567891 & 6.25345542620474 & 16.65362209505800 \\ \text { N } & 3.86718087544374 & 8.31075267694419 & 14.81686625318813 \\ \text { C } & 4.95901020677372 & 9.13709585908256 & 12.84859585584350 \\ \text { C } & 5.62727263838873 & 10.18646247752662 & 12.22671007122375 \\ \text { H } & 5.97955725022588 & 10.05783505987336 & 11.21097472578802 \\ \text { C } & 5.85166125194878 & 11.38533372288050 & 12.89076393198200 \\ \text { H } & 6.37145354792188 & 12.19122236834784 & 12.38894582542306 \\ \text { C } & 5.41784985505556 & 11.55032791324047 & 14.19959142880295 \\ \text { H } & 5.60657485130232 & 12.48302984694041 & 14.71567869348690 \\ \text { C } & 4.52476240009549 & 9.33523506783267 & 14.16895659315047 \\ \text { C } & 3.55310328616478 & 4.51716103002380 & 15.80171761762116 \\ \text { C } & 2.15643473817311 & 4.63901629536567 & 15.40366339419241 \\ \text { C } & 1.37373721019919 & 4.60958576669608 & 16.57624521708293 \\ \text { C } & 4.74660231383903 & 10.53332668332384 & 14.87092954121488 \\ \text { N } & 1.81044384428247 & 7.57600201308644 & 17.79074548763877\end{array}$




$\begin{array}{llcc}\text { C } & 3.39620634820438 & 7.48755839715877 & 15.50201904064009 \\ \text { C } & 3.60351127250658 & 4.38722859618250 & 17.21589458210562 \\ \text { C } & 2.26010201848334 & 4.49599826819064 & 17.69952854778717 \\ \text { C } & 4.71371092067055 & 4.41223534847081 & 14.85978903528713 \\ \text { H } & 4.55096573881593 & 4.99994174403049 & 13.95661427119259 \\ \text { H } & 4.87268270405492 & 3.37545368682995 & 14.54741034976110 \\ \text { H } & 5.63691998400353 & 4.76218500843569 & 15.32081980083778 \\ \text { C } & 1.64773294808728 & 4.67648941838975 & 13.99504578122569 \\ \text { H } & 0.76475643530089 & 5.30726582114245 & 13.89807847074737 \\ \text { H } & 1.37360068834208 & 3.67314713122677 & 13.65343479490903 \\ \text { H } & 2.40081932476167 & 5.05800864396198 & 13.30579069206731 \\ \text { C } & -0.11334720661066 & 4.71891389340410 & 16.67660195039733 \\ \text { H } & -0.40628498107825 & 5.43187887962655 & 17.44755190146424 \\ \text { H } & -0.55796965600573 & 3.75369195927251 & 16.93924681511817 \\ \text { H } & -0.55691568687141 & 5.04804193068062 & 15.73783818636715 \\ \text { C } & 4.83025358947815 & 4.20395581751693 & 18.05520153510962 \\ \text { H } & 5.71992244149898 & 4.56372561865928 & 17.53936345837577 \\ \text { H } & 4.99130447114437 & 3.14911862939251 & 18.29943172846189 \\ \text { H } & 4.75744380750262 & 4.75362492252598 & 18.99288635950773 \\ \text { C } & 1.81271901503091 & 4.42404479716982 & 19.12556630569835 \\ \text { H } & 2.63449743694076 & 4.60851469910310 & 19.81552586438648 \\ \text { H } & 1.39798088610389 & 3.43660412763618 & 19.35248208547864 \\ \text { H } & 1.03455411613236 & 5.15877011719562 & 19.33387920733234 \\ \text { N } & 2.95028008414304 & 7.41994251198672 & 18.12789508374516 \\ \text { O } & 3.77919874718109 & 7.77207176488915 & 18.97250691963682 \\ \text { C } & 4.72048722088379 & 7.83038342239899 & 12.14473686159737 \\ \text { H } & 5.19525181193965 & 7.00313793096574 & 12.67666207481016 \\ \text { H } & 3.65527507102456 & 7.59710738670772 & 12.07756735129844 \\ \text { H } & 5.12836714900420 & 7.85884105518818 & 11.13565834519866 \\ \mathrm{C} & 4.28367064604168 & 10.69467951776681 & 16.29096953893580 \\ \text { H } & 3.19643274508497 & 10.61517979126445 & 16.37236139068610 \\ \text { H } & 4.70019124604933 & 9.92324856905313 & 16.94160826726398 \\ \text { H } & 4.58448288365696 & 11.66553434620682 & 16.68145538987200\end{array}$




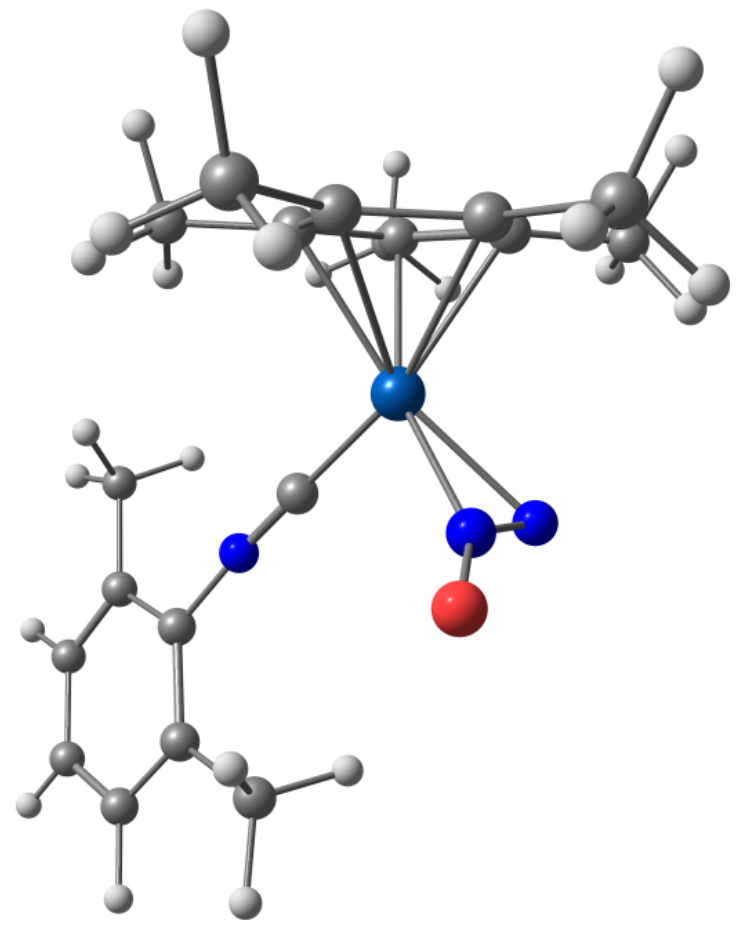

Figure S4.1. Geometry optimized structure of $\mathrm{Cp} * \mathrm{Co}\left(\eta^{2}-N, N-\mathrm{N}_{2} \mathrm{O}\right) \mathrm{CN}^{\mathrm{Xylyl}}$.
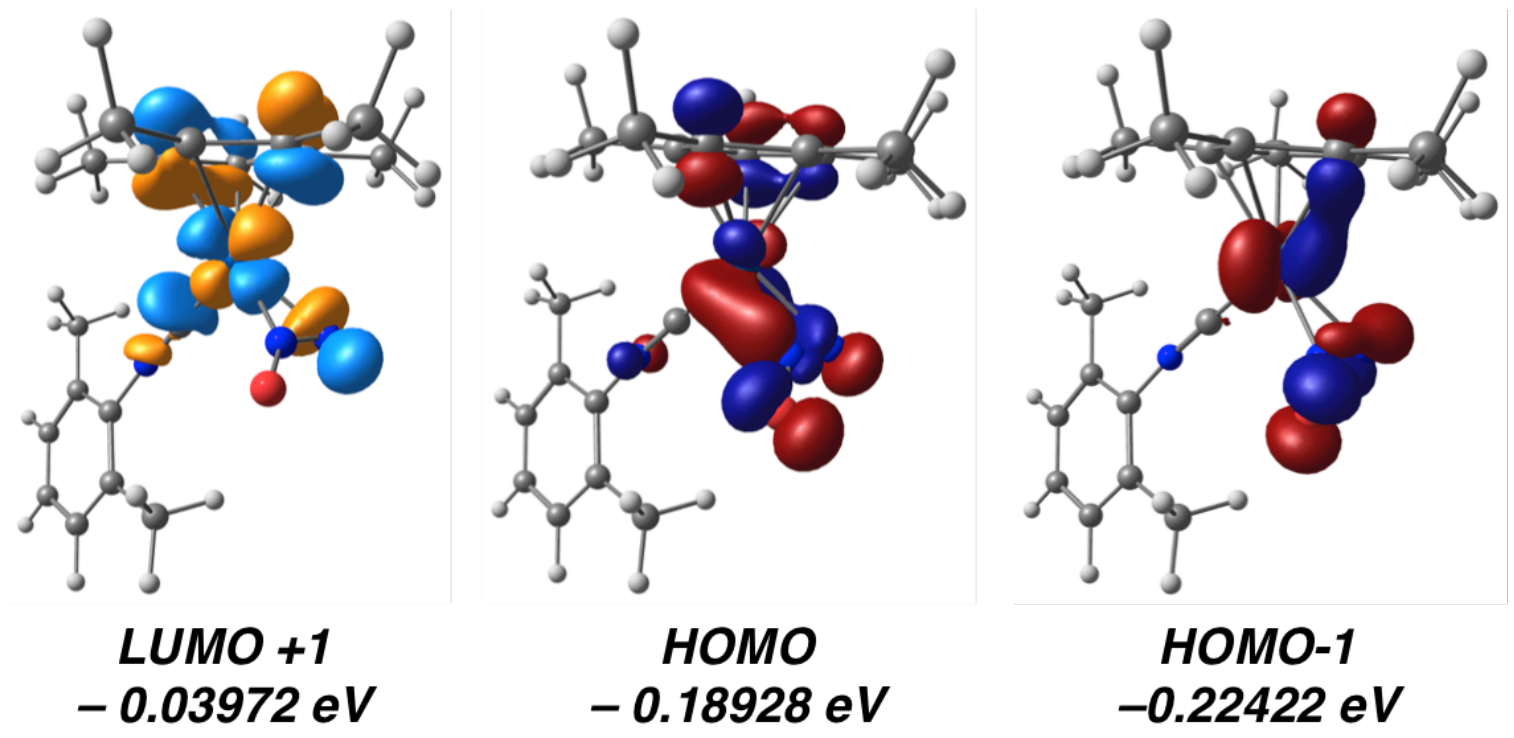

Co $d_{y z}=47.7 \%$

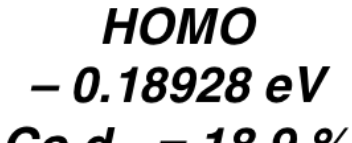

\section{HOMO-1}

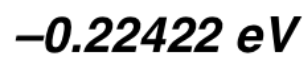

Co $d_{x 2-y 2}=49.1 \%$

Figure S4.2. Primary orbital interactions comprised of Co-d orbital character for $\mathrm{Cp} * \mathrm{Co}\left(\eta^{2}-N, N-\mathrm{N}_{2} \mathrm{O}\right) \mathrm{CN}^{\mathrm{Xylyl}}$. 


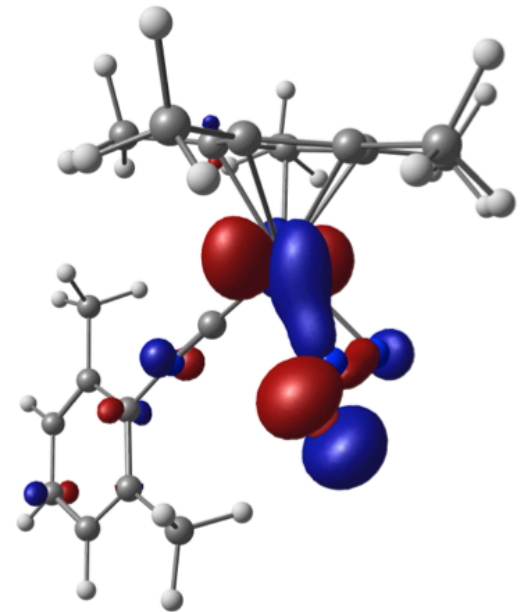

HOMO-2

$-0.23487 \mathrm{eV}$

Co $d_{x y}=47.0 \%$

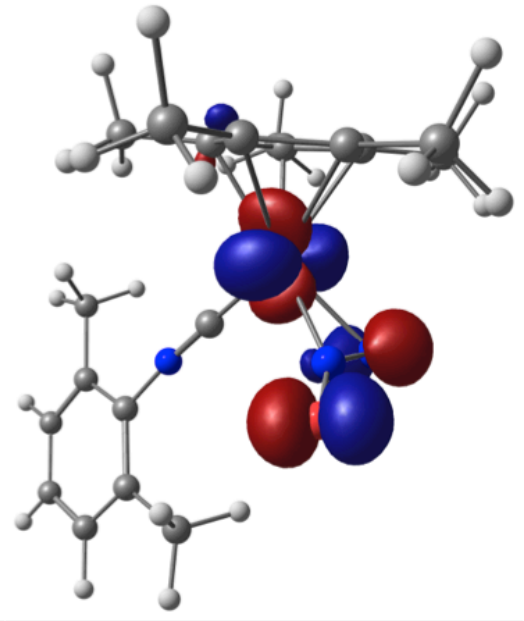

HOMO-3

$-0.24012 \mathrm{eV}$

Co $d_{z}^{2}=36.7 \%$

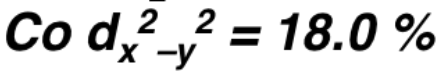

Figure S4.2 con't. Primary orbital interactions composed of Co-d orbital character $\mathrm{Cp} * \mathrm{Co}\left(\eta^{2}-N, N-\mathrm{N}_{2} \mathrm{O}\right) \mathrm{CN}^{\mathrm{Xylyl}}$.

S4.4. Mulliken Population Analysis for $\mathrm{Cp}^{*} \mathrm{Co}\left(\eta^{2}-N, N-\mathrm{N}_{2} \mathrm{O}\right) \mathrm{CN} \times \mathrm{Xyly1}$

* MULLIKEN POPULATION ANALYSIS *

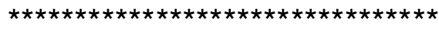

MULLIKEN ATOMIC CHARGES AND SPIN POPULATIONS

\begin{tabular}{ccc}
\hline 0 Co: & 0.179324 & 0.000000 \\
$1 \mathrm{~N}:$ & -0.059998 & 0.000000 \\
$2 \mathrm{C}:$ & 0.142193 & 0.000000 \\
$3 \mathrm{C}:$ & -0.177068 & 0.000000 \\
$4 \mathrm{H}:$ & 0.097320 & 0.000000 \\
$5 \mathrm{C}:$ & -0.103945 & 0.000000 \\
$6 \mathrm{H}:$ & 0.117715 & 0.000000 \\
$7 \mathrm{C}:$ & -0.167852 & 0.000000 \\
8 H : & 0.109340 & 0.000000 \\
$9 \mathrm{C}:$ & 0.094547 & 0.000000 \\
$10 \mathrm{C}:$ & -0.025712 & 0.000000 \\
$11 \mathrm{C}:$ & -0.088545 & 0.000000 \\
$12 \mathrm{C}:$ & 0.346028 & 0.000000 \\
$13 \mathrm{C}:$ & 0.085399 & 0.000000 \\
$14 \mathrm{~N}:$ & -0.273268 & 0.000000 \\
$15 \mathrm{C}:$ & -0.048667 & 0.000000 \\
$16 \mathrm{C}:$ & 0.278146 & 0.000000 \\
$17 \mathrm{C}:$ & -0.113347 & 0.000000 \\
$18 \mathrm{C}:$ & -0.403563 & 0.000000 \\
$19 \mathrm{H}:$ & 0.106580 & 0.000000
\end{tabular}




\begin{tabular}{|c|c|c|}
\hline & & \\
\hline & 119244 & 0000 \\
\hline & -0.399757 & \\
\hline & 7343 & 0 \\
\hline & 0.128340 & 0000 \\
\hline & 0.108624 & 0.00000 \\
\hline & -0.449054 & 0. \\
\hline & 0.149295 & 0.00000 \\
\hline & 0.123445 & 0.00000 \\
\hline & 0.109340 & 0.00000 \\
\hline & -0.440598 & 0.00000 \\
\hline & 0.111225 & 0.00000 \\
\hline & 0.123739 & 0.000000 \\
\hline & 0.139908 & 0.000000 \\
\hline & -0.403601 & 0.00000 \\
\hline & 0.124083 & 0.00000 \\
\hline & 0.126006 & 0.000000 \\
\hline 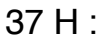 & 0.142556 & 0.000000 \\
\hline 1 & 0.240516 & 0.00000 \\
\hline & -0.411777 & 0.00000 \\
\hline & -0.402072 & 0.000000 \\
\hline & 0.126566 & 0.000000 \\
\hline & 0.133839 & 0.00000 \\
\hline & 0.118539 & 0.00000 \\
\hline & -0.385119 & 0.00000 \\
\hline & 0.144901 & 0.00000 \\
\hline & 0.15 & 0.00000 \\
\hline & 0.114120 & 0.00000 \\
\hline
\end{tabular}

Sum of atomic charges : -0.0000000

Sum of atomic spin populations: 0.0000000

\section{S4.5. Input for the geometry optimization of $\mathrm{Cp} * \mathrm{Co}\left(\eta^{2}-O, N-\mathrm{N}_{2} \mathrm{O}\right) \mathrm{CN}^{\mathrm{Xylyl}}$}

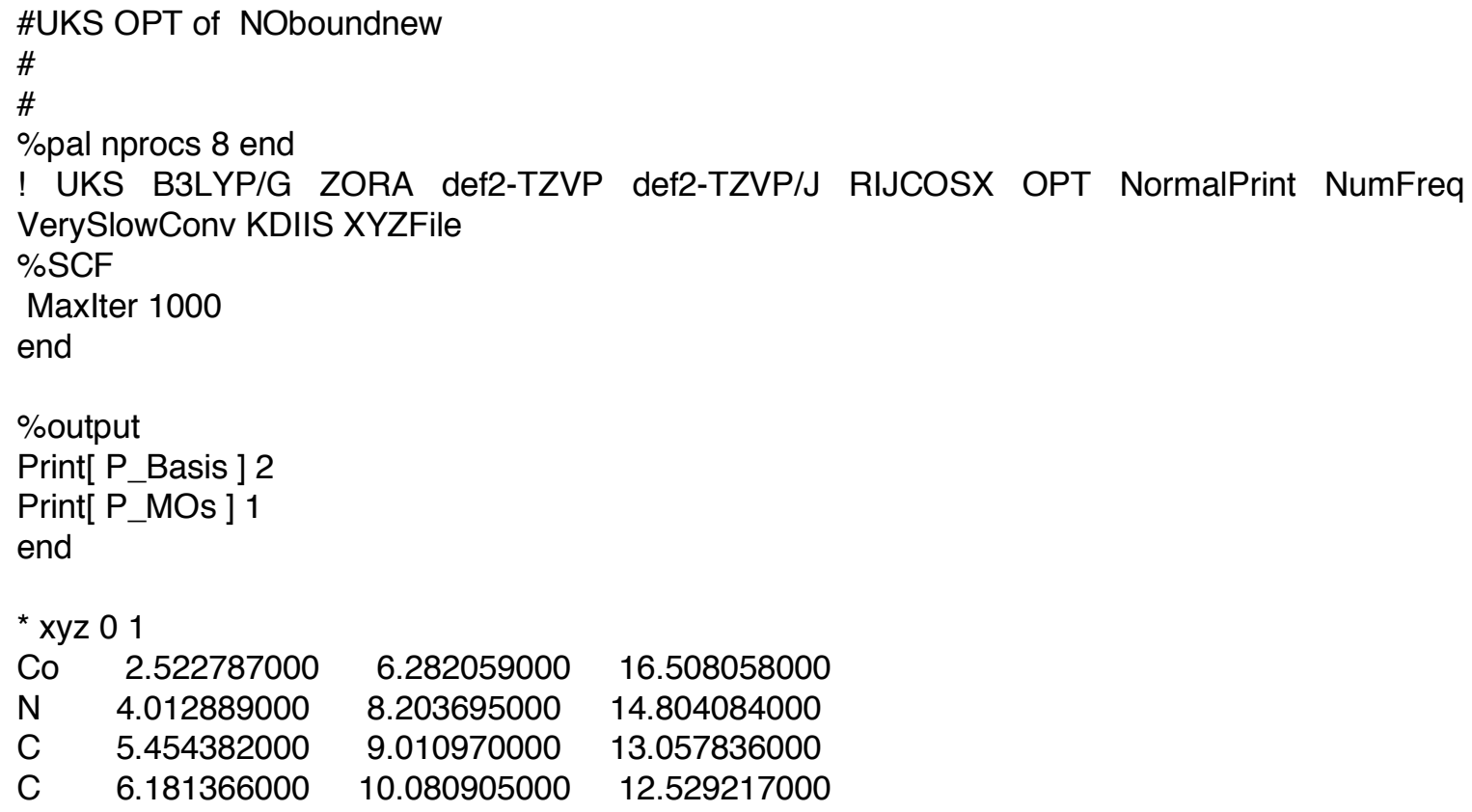




$\begin{array}{cccc}\mathrm{H} & 6.656189000 & 9.968527000 & 11.714102000 \\ \mathrm{C} & 6.217709000 & 11.297450000 & 13.176375000 \\ \mathrm{H} & 6.741334000 & 12.004501000 & 12.818072000 \\ \mathrm{C} & 5.499455000 & 11.500765000 & 14.34321000 \\ \mathrm{H} & 5.547036000 & 12.341777000 & 14.782445000 \\ \mathrm{C} & 4.740308000 & 9.246227000 & 14.234942000 \\ \mathrm{C} & 3.335159000 & 4.641406000 & 15.573633000 \\ \mathrm{C} & 1.907919000 & 4.595783000 & 15.438846000 \\ \mathrm{C} & 1.357027000 & 4.582308000 & 16.744576000 \\ \mathrm{C} & 4.711822000 & 10.492796000 & 14.878574000 \\ \mathrm{O} & 1.292423000 & 7.532094000 & 17.223565000 \\ \mathrm{C} & 3.420322000 & 7.472604000 & 15.500287000 \\ \mathrm{C} & 3.658196000 & 4.581813000 & 16.961112000 \\ \mathrm{C} & 2.436491000 & 4.557877000 & 17.682479000 \\ \mathrm{C} & 4.310592000 & 4.658925000 & 14.439596000 \\ \mathrm{H} & 3.883507000 & 5.046612000 & 13.647334000 \\ \mathrm{H} & 4.597128000 & 3.743029000 & 14.241034000 \\ \mathrm{H} & 5.090123000 & 5.198587000 & 14.687553000 \\ \mathrm{C} & 1.134764000 & 4.496158000 & 14.160265000 \\ \mathrm{H} & 0.343212000 & 5.071683000 & 14.211458000 \\ \mathrm{H} & 0.854954000 & 3.567612000 & 14.019222000 \\ \mathrm{H} & 1.700169000 & 4.783993000 & 13.413337000 \\ \mathrm{C} & -0.104816000 & 4.586951000 & 17.085487000 \\ \mathrm{H} & -0.248467000 & 5.111508000 & 17.900720000 \\ \mathrm{H} & -0.409192000 & 3.666886000 & 17.231175000 \\ \mathrm{H} & -0.611174000 & 4.985491000 & 16.347150000 \\ \mathrm{C} & 5.044981000 & 4.512604000 & 17.525026000 \\ \mathrm{H} & 5.648437000 & 5.055299000 & 16.975725000 \\ \mathrm{H} & 5.351570000 & 3.581789000 & 17.52500300 \\ \mathrm{H} & 5.041810000 & 4.855472000 & 18.443087000 \\ \mathrm{C} & 2.288920000 & 4.522261000 & 19.181635000 \\ \mathrm{H} & 3.085456000 & 4.913208000 & 19.597666000 \\ \mathrm{H} & 2.188025000 & 3.593592000 & 19.477960000 \\ \mathrm{H} & 1.497298000 & 5.036790000 & 19.444275000 \\ \mathrm{~N} & 2.183948000 & 7.453187000 & 18.033895000 \\ \mathrm{~N} & 2.546447000 & 7.629243000 & 19.260910000 \\ \mathrm{C} & 5.441107391 & 7.632085912 & 12.372195819 \\ \mathrm{H} & 6.458323018 & 7.270845347 & 12.182759616 \\ \mathrm{H} & 4.934948381 & 6.883170520 & 12.991902472 \\ \mathrm{H} & 4.919732247 & 7.670419597 & 11.408968350 \\ \mathrm{H} & 3.842379156 & 10.731470525 & 16.127054522 \\ & 2.778716929 & 10.784512646 & 15.868370057 \\ & 4.964335769 & 9.924447856 & 16.858456286 \\ & & & \\ \mathrm{H} & & \end{array}$

S4.6. Optimized coordinates for $\mathrm{Cp} * \mathrm{Co}\left(\eta^{2}-N, O-\mathrm{N}_{2} \mathrm{O}\right) \mathrm{CN}^{\mathrm{Xylyl}}$

Co 2.43298752156827

N 4.21581036779841

C 5.28828700005819

C 5.93870457219561

H 6.25943304010150

C 6.17435916923247
6.27253493729352 8.31191222414303 9.09783625783507 10.13810395401634 9.99587097234851 11.34861260220309
16.34871936075815 15.12173849142928 13.12597514291217 12.47086397095829 11.44642225596565 13.10938658576841 
H $\quad 6.67458566296514$

C 5.77113820770168

H 5.96231111072499

C 4.88565192933795

C 3.30810029799447

C 1.90535178082627

C 1.35014799068854

C 5.11981919499705

O 0.93451491679107

C 3.55923449746783

C 3.63125238001617

C 2.41848843945832

C 4.31489749017731

H 3.88596208487651

H 4.69175822487454

H 5.17572552711587

C 1.12070210712171

H 0.21366706456370

H 0.81545513646582

H 1.70313723403934

C -0.10270857173399

H -0.34040038867341

H -0.38488437856511

H -0.73193807384818

C 5.00815135591256

H 5.74263934000749

H 5.32986768504931

H 5.05226195561222

C 2.27493049104609

H 2.12779067672012

H 3.16113545855077

H 1.41839729916369

N 1.58369519537132

N 1.48859488110637

C 5.01595128356909

H 5.50099478822769

H 3.94686176590234

H 5.38443665353528

C 4.67164490704178

H 3.58656157961499

H 5.10387023492749

H 4.96896857930303
12.14902022561924

11.53555696169786

12.48041526860878

9.31731434418659

4.51492372590003

4.40425525673063

4.49777786507533

10.52668170298598

7.52085354810462

7.50560588183389

4.65914268670847

4.57377432465569

4.42644463405217

4.71557410395901

3.40394388597980

5.06764056789781

4.24760337301900

4.85232249299644

3.20810142877014

4.55470874842204

4.44236851530868

5.12463037663008

3.43404282903499

4.71425581828656

4.70616218792452

5.06577218729970

3.71073984898631

5.36252054544693

4.63543566647917

5.66081278854685

4.23523202496883

4.04769834057638

7.49809164333804

7.95773576692670

7.78943170449839

6.96019108551509

7.56595173365059

7.81070162210898

10.71280023065066

10.62421820355691

9.95453568088165

11.69250518433723
12.57949104732347

14.42471236732826

14.91695090819787

14.45352525706715

15.52104036087226

15.39784423977463

16.72337140609714

15.12760207732245

16.32921086235113

15.65603617753225

16.93180968029616

17.66551994970178

14.41637733718743

13.45758821896018

14.31444779649356

14.60510600431111

14.13154279364071

14.14672735827941

13.97388311579115

13.26319501421438

17.07746862441151

17.89326283685624

17.39669345953092

16.23161225974419

17.52223200727728

16.80186179260032

17.84580304557192

18.39101409252838

19.15244821354195

19.50761103839861

19.64647185774061

19.48501065567674

17.52194431513804

18.57764799057866

12.43979766388619

12.95697468161474

12.41687016372422

11.41532675595300

16.54898295344704

16.64348682020284

17.20478709769774

16.91903261234718 


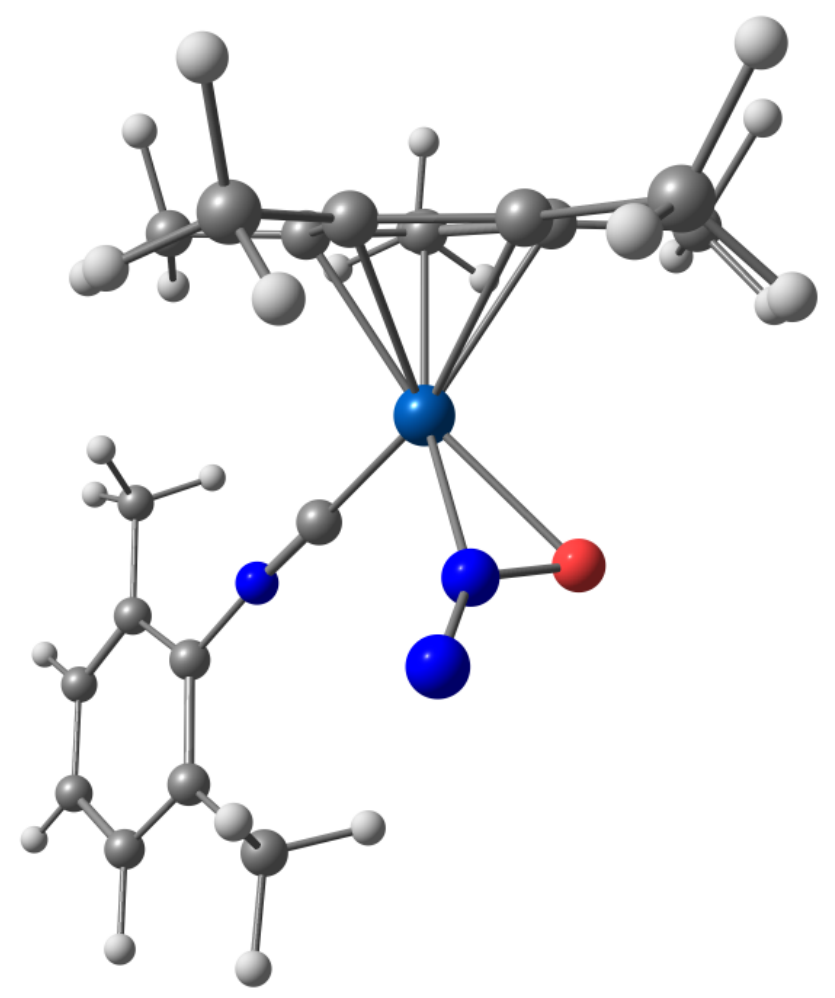

Figure S4.3. Geometry optimized structure of $\mathrm{Cp}^{*} \mathrm{Co}\left(\eta^{2}-N, O-\mathrm{N}_{2} \mathrm{O}\right) \mathrm{CN}^{\mathrm{Xylyl}}$.

\section{S4.7. Input for the geometry optimization of $\mathrm{N}_{2} \mathrm{O}$}

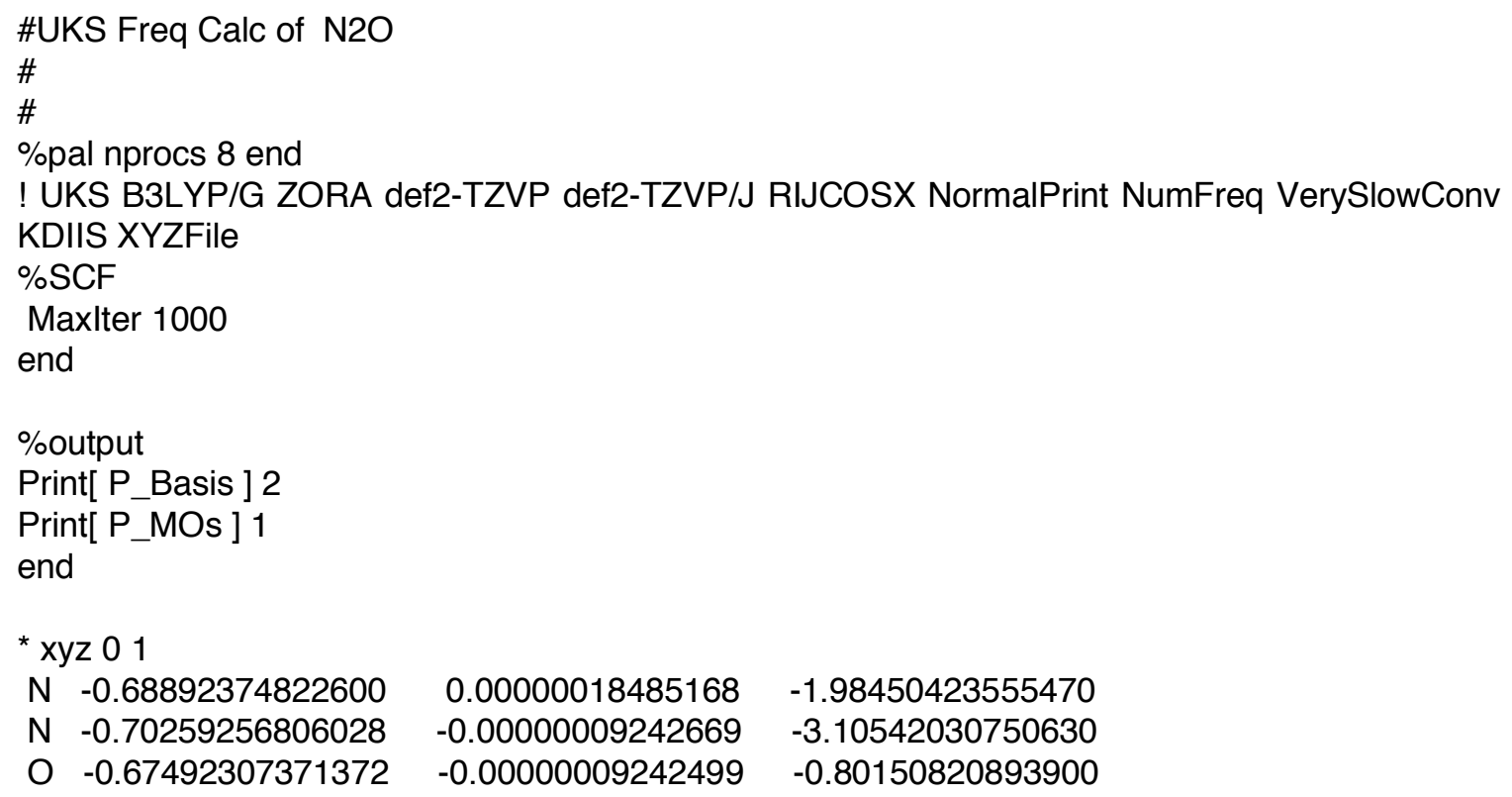




\section{S4.7. Optimized coordinates for $\mathrm{N}_{2} \mathrm{O}$}

$\begin{array}{lrrr}N & -0.68892374822600 & 0.00000018485168 & -1.98450423555470 \\ N & -0.70259256806028 & -0.00000009242669 & -3.10542030750630 \\ \mathrm{O} & -0.67492307371372 & -0.00000009242499 & -0.80150820893900\end{array}$

\section{S4.8. Mulliken Population Analysis for $\mathrm{N}_{2} \mathrm{O}$}

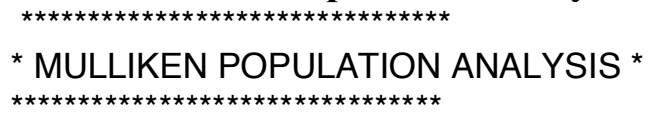

MULLIKEN ATOMIC CHARGES AND SPIN POPULATIONS

$\begin{array}{lll}0 \mathrm{~N}: & 0.430601 & 0.0000004 \\ 1 \mathrm{~N}: & -0.125505 & 0.000000 \\ 2 \mathrm{O}: & -0.305096 & 0.000000 \\ \text { Sum of atomic charges : } \quad 0.0000000 \\ \text { Sum of atomic spin populations: } \quad 0.0000000\end{array}$

\section{S5. Crystallographic Structure Determinations}

S5.1. General Information. Single crystal X-ray structure determinations were carried out at low temperature on Bruker Kappa diffractometers equipped with a Mo sealed tube or rotating anode or $\mathrm{Cu}$ rotating anode radiation source and a Bruker APEX-II, APEX-I, or Proteum Pt135 detector. All structures were solved via direct methods with SHELXS ${ }^{21}$ and refined by full-matrix least squares procedures using SHELXL ${ }^{21}$ within the Olex2 small-molecule solution, refinement and analysis software package. ${ }^{22}$ Crystallographic data collection and refinement information are listed in table S6.1.

S5.2. Information on Crystallographic Disorder: The following molecules contain positionally disordered components that were modeled and refined anisotropically. They are listed along with their respective disordered components.

OCNAr Tripp2 displays two site positional disorder in the OCN unit due to the crystallographic mirror in which it resides on. Each fragment was modeled at $50 \%$ and linked with the FVAR command and anisotropically refined.

$\left(\eta^{5}-\mathrm{Cp}^{*}\right) \mathrm{Co}\left(\eta^{2}-N, N-\mathrm{N}_{2} \mathrm{O}\right)\left(\mathrm{CNAr}^{\text {Tripp} 2}\right)$ (2) displays two site positional disorder in the $\mathrm{N}_{2} \mathrm{O}$ unit in which the oxygen is disordered over two positions. Each oxygen atom was spilt and linked with the FVAR command such that O1A and O1B are at $66.4 \%$ and $33.6 \%$ respective occupancies and anisotropically refined. It is important to note that no constraints or restraints were used in the modeling of $\mathrm{N}_{2} \mathrm{O}$ positional disorder such that the most accurate crystallographic model exhibits a large degree of thermal movement within the crystal lattice.

$\mathrm{Cp} * \mathrm{Co}\left(\eta^{2}-\mathrm{N}, \mathrm{O}-\mathrm{o}-\mathrm{CH}_{3}-\mathrm{C}_{6} \mathrm{H}_{4} \mathrm{NO}\right)\left(\mathrm{CNAr}^{\mathrm{Tripp} 2}\right) \bullet\left(\mathrm{TMS}_{2} \mathrm{O}\right)\left(\mathbf{3} \bullet\left(\mathrm{TMS}_{2} \mathrm{O}\right)\right)$ : Eight molecules of $\mathrm{TMS}_{2} \mathrm{O}$ per unit cell. 
$\mathrm{Cp} * \mathrm{Co}\left(\eta^{2}-C, C-\mathrm{C}_{6} \mathrm{H}_{10}\right) \mathrm{CNAr}{ }^{\text {Tripp2 }}$ (6) displays positional disorder in one of th $2,4,6-$ triisoprpylphenyl rings. Each ring was modeled at 50\% occupancy and linked with the FVAR command and then subjected to the EADP command. The $\mathrm{Cp}^{*}$ ring displays two site positional disorder which was modeled and refined with the AFIX command $n=10$ to generate and idealized $\mathrm{Cp}^{*}$ bond metrics. Each ring was modeled and anisotropically refined.

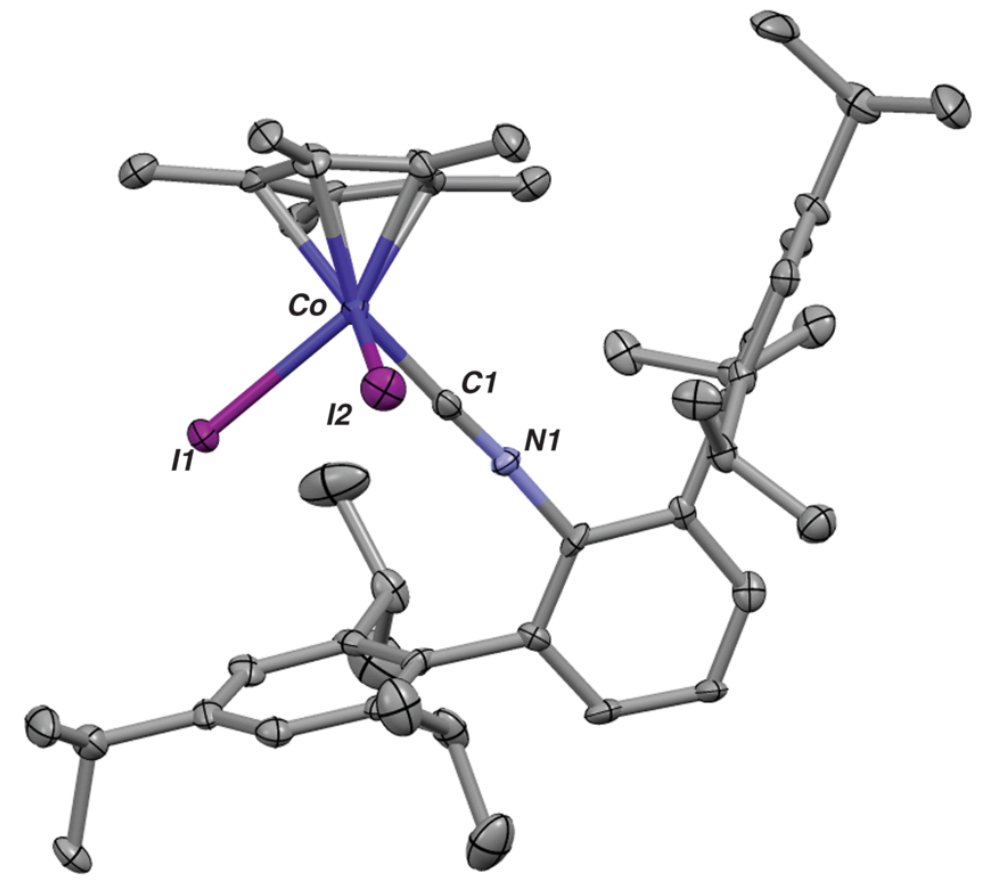

Figure S5.1. Molecular structure of $\mathrm{Cp}^{*} \mathrm{CoI}_{2} \mathrm{CNAr}{ }^{\text {Tripp2 }}$. Hydrogen atoms omitted for clarity. Selected bond distances $(\AA)$ : Co- $\mathrm{I}_{1}=2.5682(18), \mathrm{Co}_{2} \mathrm{I}_{2}=2.5944(14), \mathrm{Co}-\mathrm{C}_{1}=$ $1.837(11), \mathrm{C}_{1}-\mathrm{N}_{1}=1.136(12)$. Selected bond angles $\left({ }^{\circ}\right): \mathrm{C}_{1}-\mathrm{N}_{1}-\mathrm{C}_{2}=175.1(10)$. 


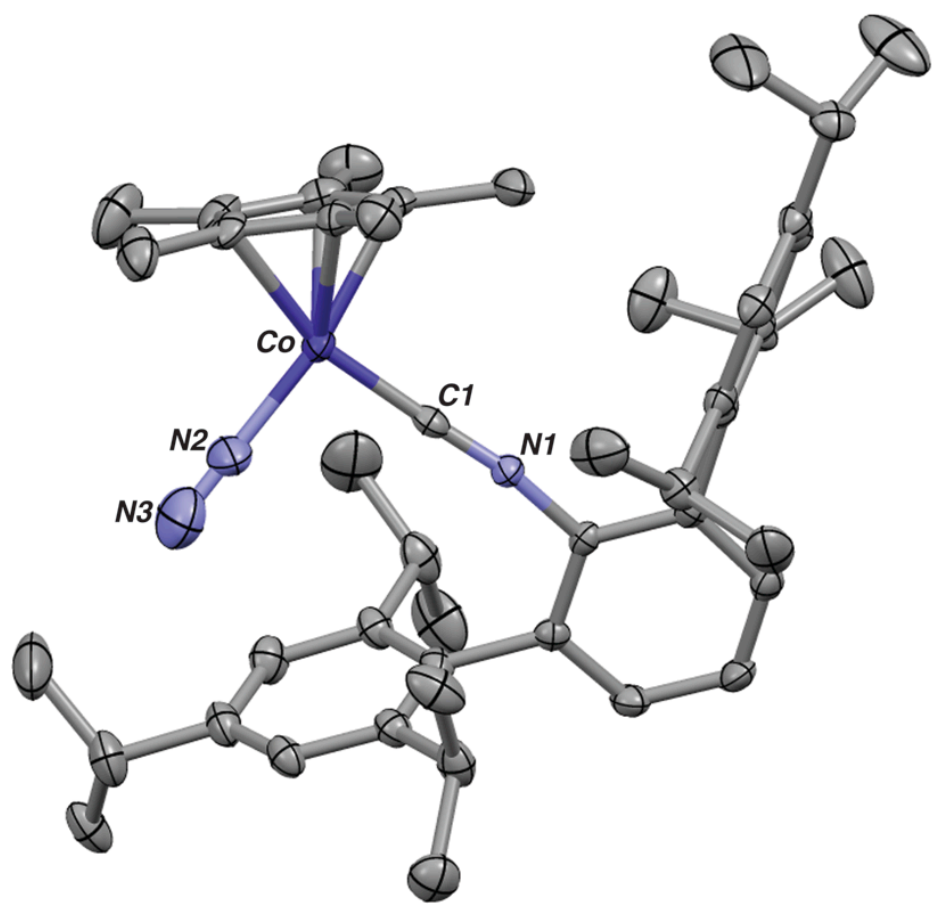

Figure S5.2. Molecular structure of $\left(\eta^{5}-\mathrm{Cp}\right) \mathrm{Co}\left(\mathrm{N}_{2}\right) \mathrm{CNAr}^{\mathrm{Tripp} 2}(\mathbf{1})$. Hydrogen atoms omitted for clarity. Selected bond distances $(\AA)$ : $\mathrm{Co}-\mathrm{C}_{1}=1.770(3), \mathrm{C}_{1}-\mathrm{N}_{1}=1.173(4)$, Co$\mathrm{N}_{2}=1.814(4), \mathrm{N}_{2}-\mathrm{N}_{3}=1.014(5)$. Selected bond angles ( $\left.{ }^{\circ}\right): \mathrm{C}_{1}-\mathrm{N}_{1}-\mathrm{C}_{2}=167.7(3), \mathrm{Co}-\mathrm{N}_{2}-$ $\mathrm{N}_{3}=176.4(5), \mathrm{C}_{1}-\mathrm{Co}-\mathrm{N}_{2}=95.50(14)$.

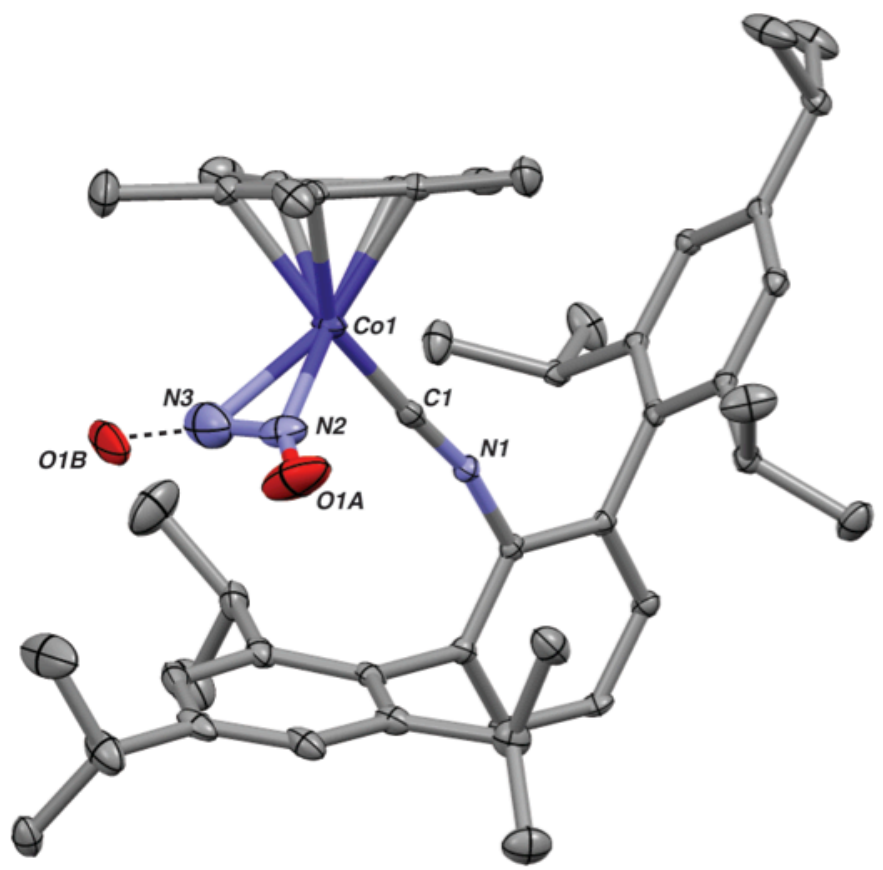

Figure S5.3. Molecular structure of $\left(\eta^{5}-\mathrm{Cp}^{*}\right) \mathrm{Co}\left(\eta^{2}-N, N-\mathrm{N}_{2} \mathrm{O}\right)\left(\mathrm{CNAr}{ }^{\mathrm{Tripp} 2}\right)$ (2). Hydrogen atoms omitted for clarity. Positional disorder with oxygen of $\mathrm{N}_{2} \mathrm{O}$ is included. Selected bond distances $(\AA)$ : Co- $\mathrm{N}_{2}=1.904(5), \mathrm{Co}-\mathrm{N}_{2}=1.952(5), \mathrm{O}_{1 \mathrm{~A}}-\mathrm{N}_{2}=1.12(1), \mathrm{O}_{1 \mathrm{~B}}-\mathrm{N}_{3}=$ 1.26(1). Selected bond angles $\left({ }^{\circ}\right)$ : $\mathrm{O}_{1 \mathrm{~A}}-\mathrm{N}_{2}-\mathrm{N}_{3}=135.2(6), \mathrm{O}_{1 \mathrm{~B}}-\mathrm{N}_{3}-\mathrm{N}_{2}=146.5(8)$. 


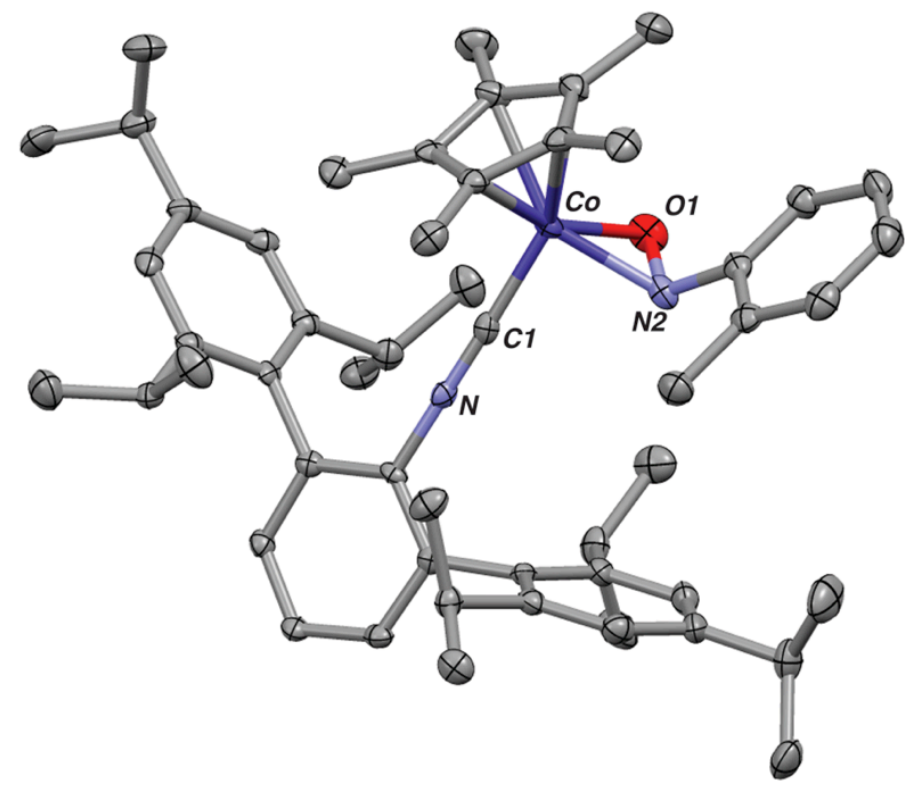

Figure S5.4. Molecular structure of $\mathrm{Cp}^{*} \mathrm{Co}\left(\eta^{2}-N, O-o-\mathrm{CH}_{3}-\mathrm{C}_{6} \mathrm{H}_{4} \mathrm{NO}\right) \mathrm{CNAr}^{\text {Tripp2 }}$ (3). Hydrogen atoms and one molecule of $\mathrm{TMS}_{2} \mathrm{O}$ of solvation omitted for clarity. Selected bond distances $(\AA)$ : Co- $\mathrm{C}_{1}=1.818(3), \mathrm{C}_{1}-\mathrm{N}_{1}=1.179(4), \mathrm{Co}_{2} \mathrm{~N}_{2}=1.945(3), \mathrm{Co}-\mathrm{O}_{1}=$ $1.908(2), \mathrm{N}_{2}-\mathrm{O}_{1}=1.371(3)$. Selected bond angles $\left({ }^{\circ}\right): \mathrm{C}_{1}-\mathrm{N}_{1}-\mathrm{C}_{2}=179.0(3), \mathrm{O}_{1}-\mathrm{N}_{2}-\mathrm{C}_{48}=$ 124.1(2).

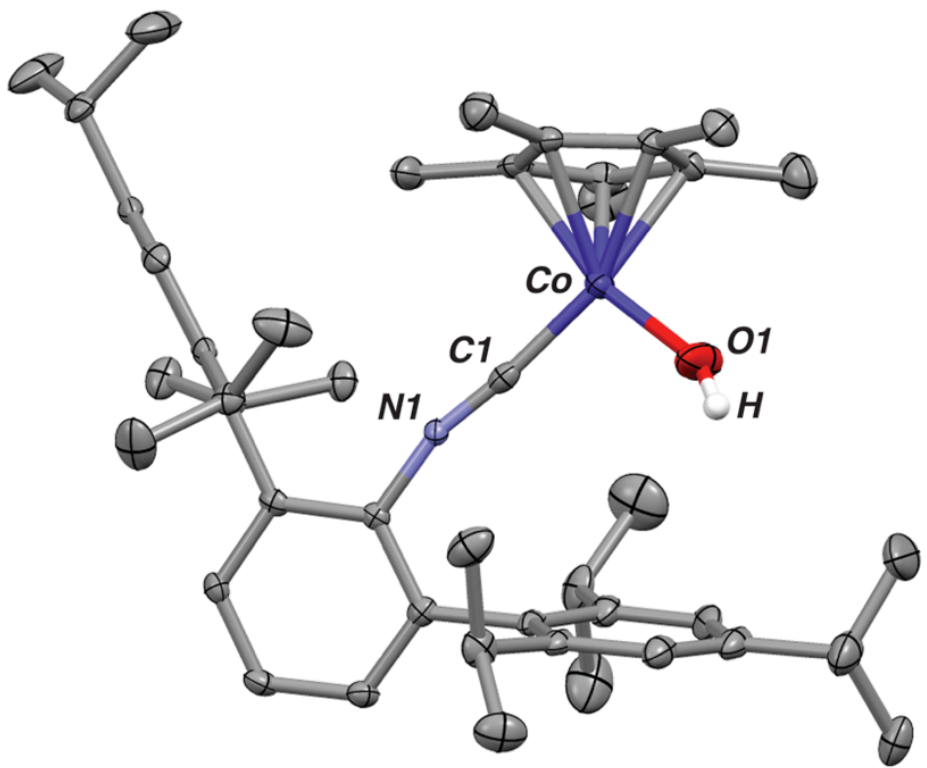

Figure S5.5. Molecular structure of $\mathrm{Cp}^{*} \mathrm{Co}(\mathrm{OH})\left(\mathrm{CNAr}^{\text {Tripp2}}\right)(4)$. Hydrogen atoms and positional disorder omitted for clarity. Selected bond distances $(\AA)$ : $\mathrm{O}_{1}-\mathrm{C}_{1}=1.172(4), \mathrm{C}_{1}$ $\mathrm{N}_{1}=1.1334\left(3\right.$. Selected bond angles $\left({ }^{\circ}\right)$ : $\mathrm{C}_{1}-\mathrm{N}_{1}-\mathrm{C}_{2}=146.27(16), \mathrm{O}_{1}-\mathrm{C}_{1}-\mathrm{N}_{1}=174.0(3)$. 


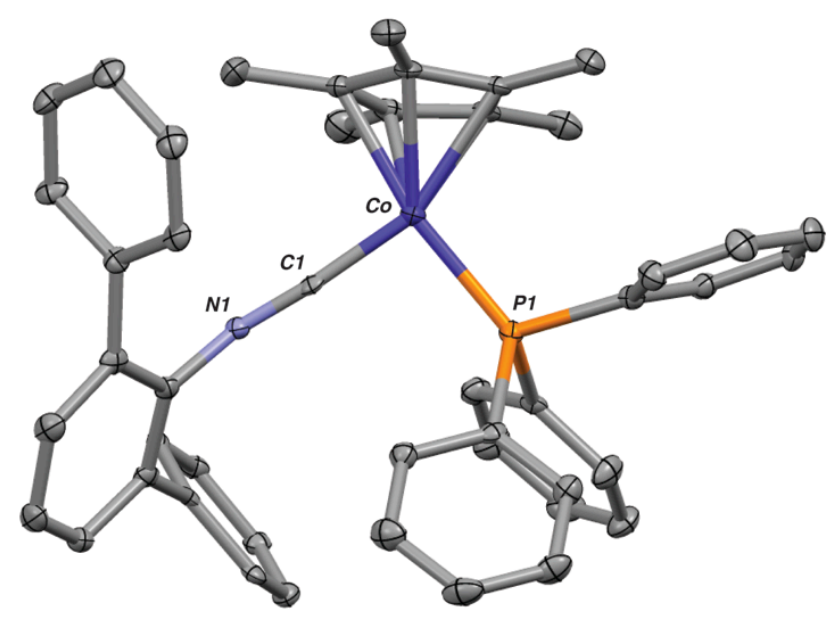

Figure S5.6. Molecular structure of $\mathrm{Cp} * \mathrm{Co}\left(\mathrm{PPh}_{3}\right) \mathrm{CNAr}^{\mathrm{Tripp} 2}(5)$. Hydrogen atoms and flanking $i$-Pr groups omitted for clarity. Selected bond distances $(\AA)$ : $\mathrm{Co}_{1}-\mathrm{P}_{1}=2.1472(10)$, $\mathrm{Co}_{1}-\mathrm{C}_{1}=1.738(4), \mathrm{C}_{1}-\mathrm{N}_{1}=1.204(4)$. Selected bond angles $\left({ }^{\circ}\right): \mathrm{C}_{1}-\mathrm{N}_{1}-\mathrm{C}_{2}=151.5(3)$.

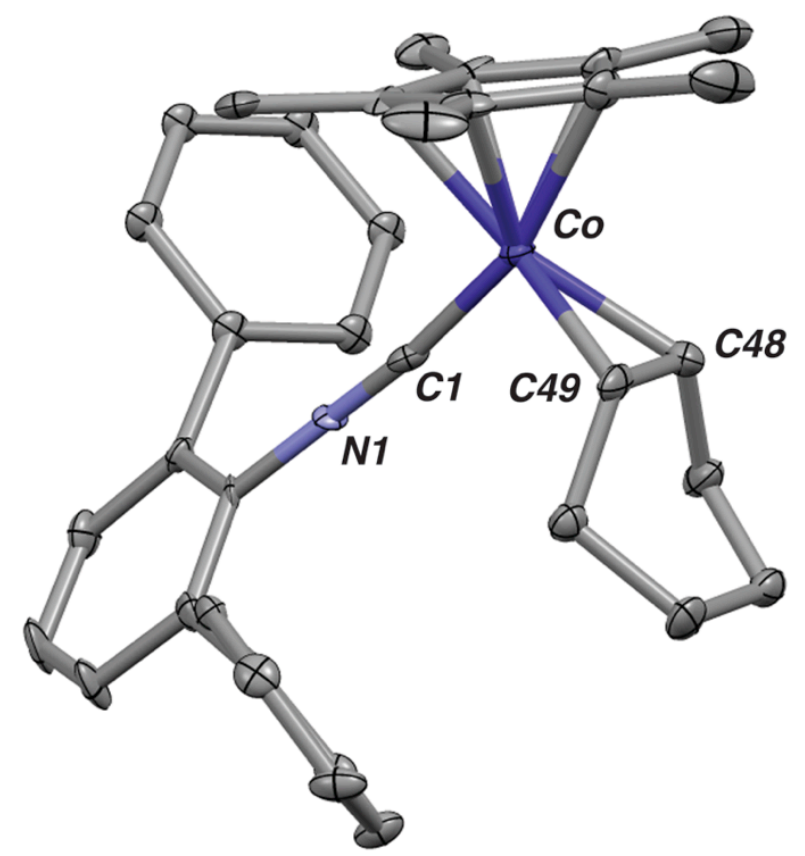

Figure S5.7. Molecular structure of $\mathrm{Cp} * \mathrm{Co}\left(\eta^{2}-C, C-\mathrm{C}_{6} \mathrm{H}_{10}\right) \mathrm{CNAr}{ }^{\text {Tripp2 }}(\mathbf{6})$. Hydrogen atoms and positional disorder omitted for clarity. Selected bond distances $(\AA): \mathrm{O}_{1}-\mathrm{C}_{1}=$ 1.172(4), $\mathrm{C}_{1}-\mathrm{N}_{1}=1.1334\left(3\right.$. Selected bond angles $\left({ }^{\circ}\right): \mathrm{C}_{1}-\mathrm{N}_{1}-\mathrm{C}_{2}=146.27(16), \mathrm{O}_{1}-\mathrm{C}_{1}-\mathrm{N}_{1}=$ 174.0(3). 


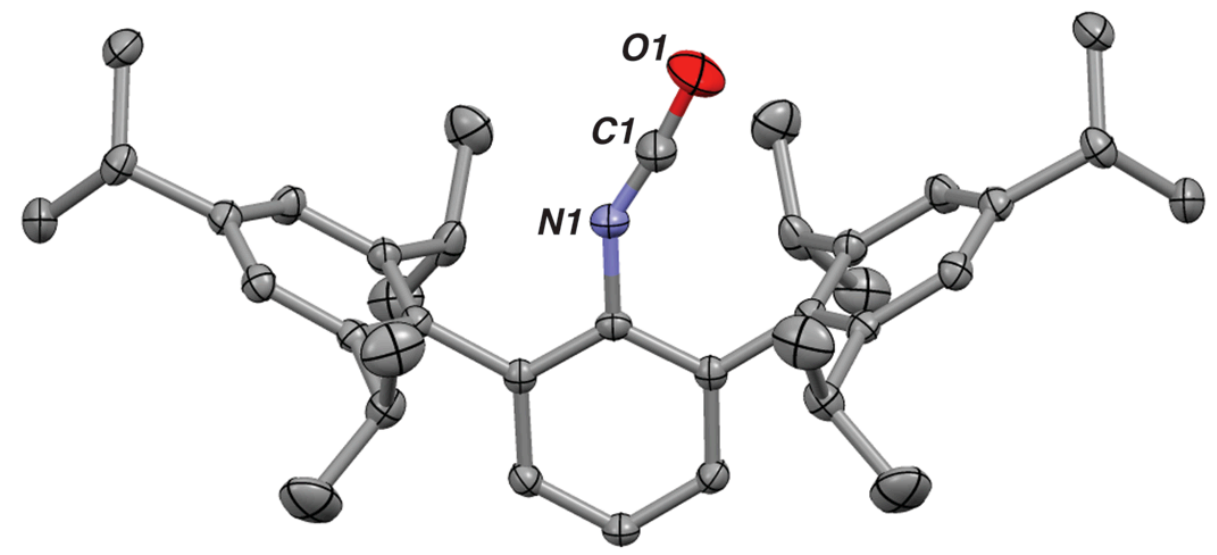

Figure S5.8. Molecular structure of OCNAr ${ }^{\text {Tripp2 }}$. Hydrogen atoms and positional disorder omitted for clarity. Selected bond distances $(\AA)$ : $\mathrm{O}_{1}-\mathrm{C}_{1}=1.172(4), \mathrm{C}_{1}-\mathrm{N}_{1}=1.1334(3$. Selected bond angles $\left({ }^{\circ}\right)$ : $\mathrm{C}_{1}-\mathrm{N}_{1}-\mathrm{C}_{2}=146.27(16), \mathrm{O}_{1}-\mathrm{C}_{1}-\mathrm{N}_{1}=174.0(3)$.

Table S5.1. Crystallographic Determination and Refinement Data

\begin{tabular}{|c|c|c|c|}
\hline Name & $\left(\eta^{5}-\mathrm{Cp}^{*}\right) \mathrm{CoI}_{2} \mathrm{CNAr}{ }^{\text {Tripp} 2}$ & $\begin{array}{c}\left(\eta^{5}-\mathrm{Cp}^{*}\right) \mathrm{Co}\left(\mathrm{N}_{2}\right) \mathrm{CNAr}^{\mathrm{Tripp} 2} \\
(\mathbf{1})\end{array}$ & $\begin{array}{c}\left(\eta^{5}-\mathrm{Cp}^{*}\right) \mathrm{Co}\left(\eta^{2}-N, N-\right. \\
\left.\mathrm{N}_{2} \mathrm{O}\right)\left(\mathrm{CNAr}{ }^{\text {Tripp} 2}\right) \\
(\mathbf{2})\end{array}$ \\
\hline Formula & $\mathrm{C}_{47} \mathrm{H}_{64} \mathrm{CoI}_{2} \mathrm{~N}$ & $\mathrm{C}_{47} \mathrm{H}_{64} \mathrm{CoN}_{3}$ & $\mathrm{C}_{47} \mathrm{H}_{64} \mathrm{CoN}_{3} \mathrm{O}$ \\
\hline Crystal System & Orthorhombic & Orthorhombic & Orthorhombic \\
\hline Space Group & Pca2 1 & Pbca & Pbca \\
\hline$a, \AA$ & $23.0483(13)$ & $17.4002(4)$ & $17.4228(7)$ \\
\hline$b, \AA$ & $14.0290(9)$ & $20.7307(5)$ & $20.6348(9)$ \\
\hline$c, \AA$ & $27.3737(18)$ & $23.4789(6)$ & $23.4337(11)$ \\
\hline$\alpha, \operatorname{deg}$ & 90 & 90 & 90 \\
\hline$\beta, \operatorname{deg}$ & 90 & 90 & 90 \\
\hline$\gamma, \operatorname{deg}$ & 90 & 90 & 90 \\
\hline $\mathrm{V}, \AA^{3}$ & $8851.1(10)$ & $8469.3(4)$ & $8392.4(6)$ \\
\hline$Z$ & 8 & 8 & 8 \\
\hline Radiation $(\lambda, \AA)$ & $\operatorname{MoK}_{\alpha}(\lambda=0.71073)$ & $\operatorname{MoK}_{\alpha}(\lambda=0.71073)$ & $\operatorname{MoK}_{\alpha}(\lambda=0.71073)$ \\
\hline$\rho$ (calcd.), $\mathrm{g} / \mathrm{cm}^{3}$ & 1.434 & 1.145 & 1.181 \\
\hline$\mu(\mathrm{Mo} \mathrm{Ka}), \mathrm{mm}^{-1}$ & 1.814 & 0.439 & 0.446 \\
\hline Temp, K & 100.0 & 100.0 & 100.0 \\
\hline$\theta$ max, deg & 50.762 & 51.524 & 50.744 \\
\hline data/parameters & $15163 / 929$ & $8045 / 477$ & $7687 / 496$ \\
\hline$R_{l}$ & 0.0567 & 0.0878 & 0.0743 \\
\hline$w R_{2}$ & 0.0897 & 0.1613 & 0.1637 \\
\hline GOF & 1.034 & 1.082 & 1.062 \\
\hline
\end{tabular}


Table S5.1. Crystallographic Determination and Refinement Data Continued.

\begin{tabular}{|c|c|c|c|}
\hline Name & $\begin{array}{c}\left(\eta^{5}-\mathrm{Cp}^{*}\right) \mathrm{Co}\left(\eta^{2}-\mathrm{N}, \mathrm{O}-\mathrm{O}-\right. \\
\left.\mathrm{CH}_{3} \mathrm{C}_{6} \mathrm{H}_{4} \mathrm{NO}\right)\left(\mathrm{CNAr}^{\mathrm{Tripp} 2}\right)^{\bullet} \\
\mathrm{TMS}_{2} \mathrm{O} \\
(\mathbf{3})\end{array}$ & $\begin{array}{c}\left(\eta^{5}-\mathrm{Cp}^{*}\right) \\
\mathrm{Co}(\mathrm{OH})\left(\mathrm{CNAr}{ }^{\text {Tripp2}}\right) \\
(\mathbf{4})\end{array}$ & $\begin{array}{c}\left(\eta^{5}-\mathrm{Cp}^{*}\right) \\
\mathrm{Co}\left(\mathrm{PPh}_{3}\right)\left(\mathrm{CNAr}{ }^{\text {Tripp2}}\right) \\
(\mathbf{5})\end{array}$ \\
\hline Formula & $\mathrm{C}_{60} \mathrm{H}_{89} \mathrm{CoN}_{2} \mathrm{O}_{2} \mathrm{Si}_{2}$ & $\mathrm{C}_{47} \mathrm{H}_{65} \mathrm{CoNO}$ & $\mathrm{C}_{65} \mathrm{H}_{79} \mathrm{CoNP}$ \\
\hline Crystal System & Orthorhombic & Orthorhombic & Monoclinic \\
\hline Space Group & $\mathrm{Pbca}$ & $\mathrm{Pbca}$ & $P 2_{1} / C$ \\
\hline$a, \AA$ & $18.4221(6)$ & $17.3101(7)$ & $13.5984(11)$ \\
\hline$b, \AA$ & $17.1331(4)$ & $20.4836(8)$ & $17.6290(12)$ \\
\hline$c, \AA$ & $36.3469(10)$ & $23.4544(11)$ & $23.0085(18)$ \\
\hline$\alpha, \operatorname{deg}$ & 90 & 90 & 90 \\
\hline$\beta, \operatorname{deg}$ & 90 & 90 & $90.367(5)$ \\
\hline$\gamma, \operatorname{deg}$ & 90 & 90 & 90 \\
\hline $\mathrm{V}, \AA^{3}$ & $11472.1(6)$ & $8316.3(6)$ & $5515.6(7)$ \\
\hline Z & 8 & 8 & 4 \\
\hline Radiation $(\lambda, \AA)$ & $\mathrm{CuK}_{\alpha}(\lambda=1.54178)$ & $\operatorname{MoK}_{\alpha}(\lambda=0.71073)$ & $\operatorname{MoK}_{\alpha}(\lambda=0.71073)$ \\
\hline$\rho$ (calcd.), g/cm ${ }^{3}$ & 1.141 & 1.148 & 1.161 \\
\hline$\mu(\mathrm{Mo} / \mathrm{Cu} \mathrm{Ka}), \mathrm{mm}^{-1}$ & 3.0051 & 0.447 & 0.380 \\
\hline Temp, K & 100.0 & 100 & 100 \\
\hline$\theta \max , \mathrm{deg}$ & 59.007 & 50.8 & 50.654 \\
\hline data/parameters & $8238 / 628$ & $7611 / 472$ & $10028 / 630$ \\
\hline$R_{I}$ & 0.0879 & 0.0593 & 0.1271 \\
\hline$w R_{2}$ & 0.1047 & 0.1408 & 0.1287 \\
\hline GOF & 1.022 & 1.022 & 1.00 \\
\hline
\end{tabular}

Table S5.1. Crystallographic Determination and Refinement Data Continued.

\begin{tabular}{|c|c|c|}
\hline Name & $\begin{array}{c}\left(\eta^{5}-\mathrm{Cp}^{*}\right) \mathrm{Co} \\
\left.\left(\eta^{2}-C, C-\mathrm{C}_{6} \mathrm{H}_{10}\right) \mathrm{CNAr}^{\mathrm{Tripp} 2}\right) \\
(\mathbf{6}) \\
\end{array}$ & OCNAr ${ }^{\text {Tripp2 }}$ \\
\hline Formula & $\mathrm{C}_{53} \mathrm{H}_{74} \mathrm{CoN}$ & $\mathrm{C}_{37} \mathrm{H}_{49} \mathrm{NO}$ \\
\hline Crystal System & Monoclinic & Orthorhombic \\
\hline Space Group & $P 2_{1} / n$ & $P b c m$ \\
\hline$a, \AA$ & $17.8319(19)$ & $10.9033(9)$ \\
\hline$b, \AA$ & $13.874(2)$ & $12.0359(11)$ \\
\hline$c, \AA$ & $19.914(3)$ & $25.140(2)$ \\
\hline$\alpha, \operatorname{deg}$ & 90 & 90 \\
\hline$\beta, \operatorname{deg}$ & $105.982(6)$ & 90 \\
\hline$\gamma, \operatorname{deg}$ & 90 & 90 \\
\hline $\mathrm{V}, \AA^{3}$ & $4737.7(11)$ & $3299.2(5)$ \\
\hline Z & 4 & 4 \\
\hline Radiation $(\lambda, \AA)$ & $\operatorname{MoK}_{\alpha}(\lambda=0.71073)$ & $\operatorname{MoK}_{\alpha}(\lambda=0.71073)$ \\
\hline$\rho$ (calcd.), $\mathrm{g} / \mathrm{cm}^{3}$ & 1.099 & 1.054 \\
\hline$\mu(\mathrm{Mo} \mathrm{Ka}), \mathrm{mm}^{-1}$ & 0.396 & 0.062 \\
\hline Temp, $\mathrm{K}$ & 100 & 100.0 \\
\hline$\theta$ max, deg & 50.024 & 50.852 \\
\hline data/parameters & $8321 / 524$ & $3120 / 196$ \\
\hline$R_{I}$ & 0.01454 & 0.0572 \\
\hline$w R_{2}$ & 0.3041 & 0.1223 \\
\hline GOF & 1.365 & 1.040 \\
\hline
\end{tabular}




\section{S6. References}

1. A. B. Pangborn, M. A. Giardello, R. H. Grubbs, R. K Rosen, and F. J. Timmers, Organometallics. 1996, 15, 1518.

2. W. L. F. Armarego and C. L. L. Chai, Purification of Laboratory Chemicals, $5^{\text {th }}$ Ed.; Elsevier, Amsterdam, 2003.

3. M. A. Schwindt, T. Lejon and L. S. Hegedus, Organometallics, 1990, 9, 2814.

4. A. E. Carpenter, C. C. Mokhtarzadeh, D. S. Ripatti, I. Havrylyuk, R. Kamezawa, C. E. Moore, A. L. Rheingold, J. S. Figueroa. Inorg. Chem. 2015, 54, 2936.

5. B. Sun, T. Yoshino, S. Matsunaga and M. Kanai, Adv. Synth. Catal., 2014, $356,1491$.

6. P. Bertani, J. Raya, B. Bechinger. Solid State Nuc. Mag. Res. 2014, 61-62, 15.

7. H. V. Le, B. Ganem. Org. Lett. 2011, 13, 2584-2585.

8. M. K. Kesharwani, B. Brauer, J. M. L. Martin. J. Phys Chem. A. 2015, 119, 1701.

9. $\quad$ S. Döring, G. Erker. Synthesis, 2001, 1, 43-45.

10. 6. F. Neese, Wiley Interdiscip. Rev.: Comput. Mol. Sci. 2012, 2, 73

11. A. D. Becke, J. Chem. Phys. 1986, 84, 4524.

12. A. D. Becke, J. Chem. Phys. 1993, 98, 5648.

13. Lee, C. T.; Yang, W. T.; Parr, R. G. Phys. Rev. B. 1988, 37, 785

14. A. Schaefer, H. Horn and R. Ahlrichs. J. Chem. Phys. 1992, 97, 2571.

15. F. Weigend and R. Ahlrichs, Phys. Chem. Chem. Phys. 2005, 7, 3297.

16. H.-J. Werner, F. R. Manby and P. J. Knowles. J. Chem. Phys. 2003, 118, 8149.

17. E. van Lenthe, E. J. Baerends and J. G. Snijders, J. Chem. Phys. 1993, 99, 4597.

18. E. van Lenthe, J. G. Snijders and E. J. Baerends, J. Chem. Phys. 1996, 105, 6505.

19. D. A. Pantazis, X. Y. Chen, C. R. Landis and F. Neese, J. Chem. Theory Comput. 2008, 4, 908 .

20. ChemCraft. G. A. Zhurko and D. A. Zhurko. 2014, www.chemcraftprog.com

21. G. M. Sheldrick, Acta Crystallogr. A., 2008, 64, 112.

22. O. V. Dolomanov, L. J. Bourhis, R. J. Gildea, J. A. K. Howard, and H. Puschmann, J. Appl. Cryst. 2009, 42, 339. 